\title{
Lectures on Classical Integrable Systems and Gauge Field Theories
}

M. Olshanetsky

In these lectures we consider Hitchin integrable systems and their relations with the self-duality equations and twisted super-symmetric Yang-Mills theory in four dimension. We define the Symplectic Hecke correspondence between different integrable systems. As an example we consider Elliptic Calogero-Moser system and integrable Euler-Arnold top on coadjoint orbits of the group GL(N, $\mathbb{C})$ and explain the Symplectic Hecke correspondence for these systems.

Keywords: Integrable systems, gauge theories, monopoles.

\section{Introduction}

Some interrelations between classical integrable systems and field theories in dimensions 3 and 4 were proposed by $\mathrm{N}$. Hitchin twenty years ago $[1,2]$. This approach to integrable systems has some advantages. It immediately leads to the Lax representation with a spectral parameter, to prove in some cases the algebraic integrability and to find separated variables [3, 4]. It was found later that some well-known integrable systems can be derived in this way $[5,6,7,8,9,10$, $11,12]$.

It was demonstrated in [13] that there exists an integrable regime in $\mathcal{N}=2$ supersymmetric Yang-Mills theory in four dimension, which is described by Sieberg and Witten [14]. A general picture of the interrelations between integrable models and gauge theories in dimensions 4, 5 and 6 was presented in [15].

Some new aspects of the interrelations between integrable systems and gauge theories have recently recently been found in the framework of four-dimensional reformulation of the geometric Langlands program [16, 17, 18]. These lectures take into account this approach, but they are also based on [1, 2, 11, 12, 19, 20, 21, 22].

The derivation of integrable systems from field theories is based on symplectic or Poisson reduction. This construction is familiar in gauge field theories. The physical degrees of freedom in gauge theories are defined upon imposing first and second the class constraints. First class constraints are analogs of the Gauss law generating gauge transformations. A combination of the Gauss law and constraints coming from the gauge fixing yields second class constraints.

We start with gauge theories that have some important properties. First, they have at least a finite number of independent conserved quantities. After reduction they will play the role of integrals of motion. Next, we assume that after gauge fixing and solving the constraints, the reduced phase space becomes a finite-dimensional manifold and its dimension is twice the number of integrals. This property provides complete integrability. It is, for example, the theory of Higgs bundles describing Hitchin integrable systems [1]. This theory corresponds to a gauge theory in three dimension. On the other hand, a similar type of constraints arises in reducing the self-duality equations in four-dimensional Yang-Mills theory [1], and in four-dimensional $\mathcal{N}=4$ supersymmetric
Yang-Mills theory [16] after reducing them to a space of dimension two.

We also analyze the problem of classifying integrable systems. Roughly speaking two integrable systems are called equivalent, if the original field theories are gauged equivalent. We extend gauge transformations by allowing singular gauge transformations of a special kind. On the field theory side these transformations correspond to monopole configurations, or, equivalently, the inclusion of the t'Hooft operators [23, 24]. For some particular examples we establish in this way an equivalence of integrable systems of particles (Calogero-Moser systems) and integrable Euler-Arnold tops. It turns out that this equivalence is the same as equivalence of two types of $R$-matrices of dynamical and vertex type [25, 26].

Before considering concrete cases we point out the main definitions of completely integrable systems [20, 21, 27].

\section{Classical integrable systems}

Consider a smooth symplectic manifold $\mathcal{R}$ of $\operatorname{dim}(\mathcal{R})=2 l$. It means that there exists a closed non-degenerate two-form $\mathrm{w}$, and the inverse bivector $\pi\left(\omega_{a, b} \pi^{b c}=\delta_{a}^{c}\right)$, such that the space $C^{\infty}(\mathcal{R})$ becomes a Lie algebra (Poisson algebra) with respect to the Poisson brackets

$$
\{F, G\}=\langle d F|\pi| d G\rangle=\partial_{a} F \pi^{a b} \partial_{b} G .
$$

Any $H \in C^{\infty}(\mathcal{R})$ defines a Hamiltonian vector field on $\mathcal{R}$

$$
H \rightarrow\langle d H| \pi=\partial_{a} H \pi^{a b} \partial_{b}=\{H,\} .
$$

A Hamiltonian system is a triple $(\mathcal{R}, \pi, H)$ with the Hamiltonian flow

$$
\partial_{t} x^{a}=\left\{H, x^{a}\right\}=\left(\partial_{b} H\right) \pi^{b a}
$$

A Hamiltonian system is called completely integrable, if it satisfies the following conditions

- there exist $l$ Poisson commuting Hamiltonians on $\mathcal{R}$ (integrals of motion) $I_{1}, \ldots, I_{l}$.

- Since the integrals commute the set $T_{c}=\left\{\left\{I_{j}=c_{j}\right\}\right.$ is invariant with respect to the Hamiltonian flows $\left\{I_{j},\right\}$. Then being restricted on $T c, I_{j}(x)$ are functionally independent for almost all $x \in T_{c}$, i.e. $\operatorname{det}\left(\partial_{a} I_{b}\right)(x) \neq 0$. 
In this way we come to the hierarchy of commuting flows on $\mathcal{R}$

$$
\partial_{t_{j}} \mathbf{x}=\left\{I_{j}(\mathbf{x}), \mathbf{x}\right\} .
$$

$T_{c}=$ is a submanifold $T_{c} \subset \mathcal{R}$. It is is a Lagrangian submanifold, i.e. $\omega$ vanishes on $T_{c}$. If $T_{c}$ is compact and connected, then it is diffeomorphic to an $l$-dimensional torus. Torus $T_{c}$ is called the Liouville torus. In a neighborhood of $T_{C}$ there is a projection

$$
p: \mathcal{R} \rightarrow B,
$$

where the Liouville tori are generic fibers and the base of fibration $B$ is parameterized by the values of the integrals. The coordinates on a Liouville torus ("the angle" variables) along with dual variables on $B$ ("the action" variables) describe a linearized motion on the torus. Globally, the picture can be more complicated. For some values of $c_{j} T_{c}$ ceases to be a submanifold. In this way the action-angle variables are local.

Here we consider a complex analog of this picture. We assume that $\mathcal{R}$ is a complex algebraic manifold and the symplectic form $\omega$ is a $(2,0)$ form, i.e. locally in the coordinates $\left(z^{1}, \bar{z}^{1}, \ldots, z^{l}, \bar{z}^{l}\right)$ the form is represented as $\omega=\omega_{a, b} d z^{a} \wedge d z^{b}$. General fibers of (2.2) are abelian subvarieties of $\mathcal{R}$, i.e. they are complex tori $\mathbb{C}^{l} / \Lambda$, where the lattice $\Lambda$ satisfies the Riemann conditions. Integrable systems in this situation are called algebraically integrable systems.

Let two integrable systems be described by two isomorphic sets of the action-angle variables. In this case the integrable systems can be considered as equivalent. Establishing equivalence in terms of angle-action variables is troublesome. There is a more direct way based on Lax representation. Lax representation is one of the commonly accepted methods for constructing and investigating integrable systems. Let $L(x, z)$, $M_{1}(x, z), \ldots, M_{l}(x, z)$ be a set of $l+1$ matrices depending on $x \in \mathcal{R}$ with a meromorphic dependence on the spectral parameter $z \in \Sigma$, where $\Sigma$ is a Riemann surface. (It will be explained below that $L$ and $M$ are sections of some vector bundles over $\Sigma$.) This is called a basic spectral curve. Assume that the commuting flows (2.1) can be rewritten in the matrix form

$$
\partial_{t_{j}} L(\mathbf{x}, z)=\left[L(\mathbf{x}, z), M_{j}(\mathbf{x}, z)\right] .
$$

Let $f$ be a non-degenerate matrix of the same order as $L$ and $M$. The transformation

$$
L^{\prime}=f^{-1} L f, M_{j}^{\prime}=f^{-1} \partial_{t_{j}} f+f^{-1} M_{j} f,
$$

is called the gauge transformation because it preserves the Lax form (2.3). The flows (2.3) can be considered as special gauge transformations

$$
L\left(t_{1}, \ldots, t_{l}\right)=f^{-1}\left(t_{1}, \ldots, t_{l}\right) L_{0} f\left(t_{1}, \ldots, t_{l}\right),
$$

where $L_{0}$ is independent on times and defines an initial data, and $M_{j}=f^{-1} \partial_{t_{j}} f$. Moreover, it follows from this representation that the quantities $\operatorname{tr}(L(\mathbf{x}, z))^{j}$ are preserved by the flows and thereby can produce, in principle, the integrals of motion. As we mentioned above, it is reasonable to consider two integrable systems to be equivalent if their Lax matrices are related by a non-degenerate gauge transformation.

We relax the definition of the gauge transformations and assume that can have poles and zeroes on the basic spectral curve $\Sigma$ with some additional restrictions on $f$. This equivalence is called the Symplectic Hecke Correspondence. This extension of equivalence will be considered in details in these lectures. The following systems are equivalent in this sense:

\section{EXAMPLES}

- 1. Elliptic Calogero-Moser system $\Leftrightarrow$ Elliptic $\operatorname{GL}(N, \mathbb{C})$ Top, [11];

- 2. Calogero-Moser field theory $\Leftrightarrow$ Landau-Lifshitz equation, $[11,10]$;

- 3. Painlevé VI $\Leftrightarrow$ Zhukovsky-Volterra gyrostat, [12].

The first example will be considered in Section 4 .

The gauge invariance of the Lax matrices allows one to define the spectral curve

$$
\mathcal{C}=\{(\lambda \in \mathbb{C}, z \in \Sigma) \mid \operatorname{det}(\lambda-L(x, z))=0\}
$$

The Jacobian of $\mathcal{C}$ is an abelian variety of dimension $g$, where $g$ is the genus of $\mathcal{C}$. If $g=l=\frac{1}{2} \operatorname{dim} \mathcal{R}$ then $\mathcal{J}$ plays the role of the Liouville torus and the system is algebraically integrable. In generic cases $g>l$ and to prove algebraic integrability one should find additional reductions of the Jacobians, leading to abelian spaces of dimension $l$.

Finally we formulate two goals of these lectures

- to derive the Lax equation and the Lax matrices from a gauge theory;

- to explain the equivalence between integrable models by inserting t'Hooft operators in gauge theory.

\section{1d field theory}

The simplest integrable models, such as the rational Calogero-Moser system, the Sutherland model, and the open Toda model can be derived from matrix models of a finite order. Here we consider a particular case - the rational Calogero-Moser system (RCMS) [32, 33].

\subsection{Rational Calogero-Moser System (RCMS)}

The phase space of the RCMS is $\mathcal{R}^{R C M}=C^{2 N}=\{(\mathbf{v}, \mathbf{u})\}, \mathbf{v}=\left(v_{1}, \ldots, v_{N}\right), \mathbf{u}=\left(u_{1}, \ldots, u_{N}\right)$ with the canonical symplectic form

$$
\omega^{R C M}=\sum_{j=1}^{N} d v_{j} \wedge d u_{j},\left\{v_{j}, u_{k}\right\}=\delta_{j k} .
$$

The Hamiltonian describes interacting particles with complex coordinates $\mathbf{u}=\left(u_{1}, \ldots, u_{N}\right)$ and complex momenta $\mathbf{v}=\left(v_{1}, \ldots, v_{N}\right)$

$$
H^{R C M}=\frac{1}{2} \sum_{j=1}^{N} v_{j}^{2}+v^{2} \sum_{j \star} \frac{1}{\left(u_{j}-u_{k}\right)^{2}} .
$$


The Hamiltonian leads to the equations of motion

$$
\begin{aligned}
& \partial_{t} u_{j}=v_{j}, \\
& \partial_{t} v_{j}=-v^{2} \sum_{j \neq} \frac{1}{\left(u_{j}-u_{k}\right)^{3}} .
\end{aligned}
$$

The equations of motion can be put in the Lax form

$$
\partial_{t} L(\mathbf{v}, \mathbf{u})=[L(\mathbf{v}, \mathbf{u}), M(\mathbf{v}, \mathbf{u})] .
$$

Here $L, M$ are the $N \times N$ matrices of the form

$$
\begin{aligned}
& L=P+X, M=D+Y, \\
& P=\operatorname{diag}\left(v_{1}, \ldots, v_{N}\right), X_{j k}=v\left(u_{j}-u_{k}\right)^{-1}, \\
& Y_{j k}=-v\left(u_{j}-u_{k}\right)^{-2}, D=\operatorname{diag}\left(d_{1}, \ldots, d_{N}\right), \\
& d_{j}=v \sum_{k \neq j}\left(u_{j}-u_{k}\right)^{-2} .
\end{aligned}
$$

The diagonal part of the Lax equation (3.4) implies $\partial_{t} P=[X, Z]_{\text {diag }}$. It coincides with (3.3). The non-diagonal part has the form

$$
\partial_{t} X=[P, Y]+\left([X, Y]_{\text {nondiag }}-[X, D]\right) .
$$

It can be found that $[X, Y]_{\text {nondiag }}=[X, D]$ and the equation $\partial_{t} X=[P, Y]$ coincides with (3.2).

The Lax equation produces the integrals of motion

$$
I_{m}=\frac{1}{m} \operatorname{tr}\left(L^{m}\right), \partial_{t} \operatorname{tr}\left(L^{m}\right)=0, m=1,2, \ldots, N .
$$

It will be proved later that they are in involution $\left\{I_{m}, I_{n}\right\}=0$. In particular, $I_{2}=H^{R C M}$. Eventually, we come to the RCMS hierarchy

$$
\partial_{j} f(\mathbf{v}, \mathbf{u})=\left\{I_{j}, f(\mathbf{v}, \mathbf{u})\right\} .
$$

\subsection{Matrix mechanics and RCMS}

This construction was proposed in Ref. [35, 36]. Consider a matrix model with the phase space $\mathcal{R}=\operatorname{gl}(N, \mathbb{C}) \oplus \operatorname{gl}(N, \mathbb{C})$

$$
\mathcal{R}=(\Phi, \bar{A}), \Phi, \bar{A} \in \operatorname{gl}(N, \mathbb{C})
$$

(These notations will be justified in next section)

$$
\operatorname{dim} \mathcal{R}=2 N^{2} .
$$

The symplectic form on $\mathcal{R}$ is

$$
\omega=\operatorname{tr}(d \Phi \wedge d \bar{A})=\sum_{j, k} d \Phi_{j, k} \wedge d \bar{A}_{j, k} .
$$

The corresponding Poisson brackets have the form

$$
\left\{\Phi_{j, k}, \bar{A}_{j, k}\right\}=\delta_{k i} \delta_{j l} \text {. }
$$

Choose $N$ commuting integrals

$$
I_{m}=\frac{1}{m} \operatorname{tr}\left(\Phi^{m}\right),\left\{I_{m}, I_{n}\right\}=0, m=1,2, \ldots, N .
$$

Take as a Hamiltonian $H=I_{2}$. Then we come to the free motion on $\mathcal{R}$

$$
\begin{aligned}
& \partial_{t} \Phi=\{H, \Phi\}=0, \\
& \partial_{t} \bar{A}=\{H, \bar{A}\}=\Phi .
\end{aligned}
$$

Generally, we have a free matrix hierarchy

$$
\partial_{j} \Phi=0, \partial_{j} \bar{A}=\Phi^{j-1},\left(\partial_{j}=\left\{I_{j},\right\}\right) .
$$

\subsubsection{Hamiltonian reduction for RCMS}

The form $\omega$ and the integrals $I_{m}$ are invariant with resect to the action of the gauge group

$$
\begin{aligned}
& \mathcal{G}=\mathrm{GL}(N, \mathbb{C}), \\
& \Phi \rightarrow f^{-1} \Phi f, \bar{A} \rightarrow f^{-1} \bar{A} f, f \in \mathrm{GL}(N, \mathbb{C}) .
\end{aligned}
$$

The action of gauge Lie algebra $\operatorname{Lie}(\mathcal{G})=\operatorname{gl}(N, \mathbb{C})$ is represented by the vector fields

$$
V_{\epsilon} \Phi=[\Phi, \epsilon], \mathrm{V}_{\epsilon} \bar{A}=[\bar{A}, \epsilon] .
$$

Let $\iota_{\epsilon}$ be the contraction operator with respect to the vector field $V_{\epsilon}\left(\iota_{\epsilon}=\sum_{j, k}\left(V_{\epsilon}\right)_{j k} \frac{\partial}{\partial j k}\right)$ and $\mathcal{L}_{\epsilon}=\mathrm{d} \iota_{\epsilon}+\iota_{\epsilon} \mathrm{d}$ is the corresponding Lie derivative. The invariance of the symplectic form and the integrals means that

$$
\mathcal{L}_{\epsilon} \omega=0, \mathcal{L}_{\epsilon} I_{m}=0 .
$$

Since the symplectic form is closed $d \omega=0$, we have $d \iota_{\epsilon} \omega=0$. Then on the affine space $\mathcal{R}$ the one-form $\iota_{\epsilon} \omega$ is exact

$$
\iota_{\epsilon} \omega=d F(\Phi, \bar{A}, \epsilon) \text {. }
$$

The function $F(\Phi, \bar{A}, \epsilon)$ is called the momentum Hamiltonian. The Poisson brackets with the momentum Hamiltonian generate the gauge transformations:

$$
\{F, f(\Phi, \bar{A})\}=\mathcal{L}_{\epsilon} f(\Phi, \bar{A}) .
$$

The explicit form of the momentum Hamiltonian is

$$
F(\Phi, \bar{A}, \epsilon)=\operatorname{tr}(\epsilon[\Phi, \bar{A}]) .
$$

Define the moment map

$$
\begin{aligned}
& \mu: \mathcal{R} \rightarrow \text { Lie } *(\text { gauge group }) \sim \operatorname{gl}(N, \mathbb{C}) \\
& \mu(\Phi, \bar{A})=[\Phi, \bar{A}],(\Phi, \bar{A}) \mapsto[\Phi, \bar{A}] .
\end{aligned}
$$

Let us fix its value as

$$
\begin{aligned}
& \mu[\Phi, \bar{A}]=v J, \\
& J=\left(\begin{array}{ccccc}
0 & 1 & \cdots & \cdots & 1 \\
1 & 0 & 1 & \cdots & 1 \\
\vdots & \ddots & \ddots & \cdots & \vdots \\
1 & \cdots & \cdots & 1 & 0
\end{array}\right) .
\end{aligned}
$$

It follows from the definition of the moment map that (3.16) is the first class constraints. In particular, $\left\{F(\Phi, \bar{A}, \epsilon), F\left(\Phi, \bar{A}, \epsilon^{\prime}\right)\right\}=F\left(\Phi, \bar{A},\left[\epsilon, \epsilon^{\prime}\right]\right)$. Note, that matrix $J$ is degenerate and is conjugated to the diagonal matrix $\operatorname{diag}(N-1,-1, \ldots-1)$. Let $\mathcal{G}_{0}$ be a subgroup of the gauge group preserving the moment value

$$
\mathcal{G}_{0}=\left\{f \in \mathcal{G} \mid f^{-1} J f=J\right\},\left(\operatorname{dim}\left(\mathcal{G}_{0}\right)=(N-1)^{2}+1\right) .
$$

In other words $\mathcal{G}_{0}$ preserves the surface in $\mathcal{R}$

$$
F^{-1}(v J)=\{[\Phi, \bar{A}]=v J\} \text {. }
$$

Let us fix a gauge on this surface with respect to the $\mathcal{G}_{0}$ action. It can be proved that generic matrices $\bar{A}$ can be diagonalized by $\mathcal{G}_{0}$

$$
f^{-1} \bar{A} f=\mathbf{u}=\operatorname{diag}\left(u_{1}, \ldots, u_{n}\right), f \in \mathcal{G}_{0} .
$$


In other words, we have two conditions - first class constraints (3.16) and gauge fixing (3.19). The reduced phase space $\mathcal{R}^{\text {red }}$ is the result of putting both types of constraints

$$
\mathcal{R}^{\text {red }}=\mathcal{R} / / \mathcal{G}=F^{-1}(v J) / \mathcal{G}_{0} .
$$

It has dimension

$$
\left\{\begin{array}{llll}
\operatorname{dim}\left(\mathcal{R}^{\text {red }}\right) & =\operatorname{dim}(\mathcal{R}) & -\operatorname{dim}(\mathcal{G}) & -\operatorname{dim}\left(\mathcal{G}_{0}\right) \\
2 N-2 & =2 N^{2} & -N^{2} & -(N-1)^{2}+1
\end{array}\right.
$$

Let us prove that $\mathcal{R}^{\text {red }}=\mathcal{R}^{R C M}$ and that the hierarchy (3.12) being restricted to $\mathcal{R}^{R C M}$ coincides with the RCMS hierarchy (3.8). Let $f \in \mathcal{G}_{0}$ diagonalize $\bar{A}$ in (3.19). Define

$$
L=f^{-1} \Phi f
$$

Then it follows from (3.10) that $L$ satisfies the Lax equation

$$
\partial_{t} \Phi=0 \Rightarrow \partial_{t} L=[L, M],\left(M=-f^{-1} \partial_{t} f\right) .
$$

The moment constraints (3.18) allow one to find the off-diagonal part of $L$. Evidently, it coincides with $X$ (3.5). The diagonal elements of $L$ are free parameters. In a similar way, the off-diagonal part $Y$ (3.6) of $M$ can be derived from the equation of motion for $\bar{A}$ (3.11). Thereby, we come to the Lax form of the equations of motion for RCMS. Since $\Phi \rightarrow L$ and $\bar{A} \rightarrow \mathbf{u}$, the symplectic form $\omega$ (3.9) coincides on $\mathcal{R}^{R C M}$ with $\omega^{R C M}$ (3.1). It follows from (3.20) that the integrals (3.7) Poisson commute. Therefore, we obtain RCMS hierarchy.

The same system can be derived starting with matrix mechanics based on $\operatorname{SL}(N, \mathbb{C})$. In this case $I_{1}=\operatorname{tr} \Phi=0$ and thereby in the reduced system $\sum v_{j}=0$.

\subsubsection{Hamiltonian reduction for 4d Yang-Mills theory}

In this subsection we take a step aside to illustrate the Hamiltonian reduction in terms of the familiar phase space of the Yang-Mills theory. For this purpose consider 4d YM theory with a group $G$ in the Hamiltonian formalism [37, 38]. The phase space is generated by the space components on $\mathbb{R}^{3}=\left(x^{1}, x^{2}, x^{3}\right)$ of the vector potential and the electric field

$$
\mathcal{R}=\left\{\mathbf{A}=\left(A_{1}, A_{2}, A_{3}\right), \mathbf{E}=\left(E_{1}, E_{2}, E_{3}\right)\right\} .
$$

where

$$
E_{j}=F_{0 j}=\partial_{0} A_{j}-\partial_{j} A_{0}+\left[A_{0}, A_{j}\right] .
$$

Here we have suppressed the Lie algebra indices. It is a symplectic space with the canonical form

$$
\omega=\int_{\mathbb{R}^{3}}\langle d \mathbf{E} \wedge d \mathbf{A}\rangle=\sum_{j=1}^{3} \int_{\mathbb{R}^{3}} \operatorname{tr}\left(d E_{j} \wedge d A_{j}\right) .
$$

The Hamiltonian is quadratic in fields and has the form

$$
H=\frac{1}{2} \int_{\mathbb{R}^{3}}\left\langle\mathbf{E}^{2}\right\rangle+\left\langle\mathbf{B}^{2}\right\rangle,
$$

where $\mathbf{B}=\left(B_{1}, B_{2}, B_{3}\right)$ is the magnetic field

$$
B_{j}=\epsilon_{j k l} F_{k l}=\epsilon_{j k l}\left(\partial_{k} A_{j}-\partial_{j} A_{k}+\left[A_{k}, A_{j}\right]\right) .
$$

We assume that the fields are smooth and vanish at infinity such that the Hamiltonian and the symplectic form are well defined. The Hamiltonian defines the classical equations of motion

$$
\partial_{t} A_{j}=E_{j}, \partial_{t} E_{j}=\sum_{k}\left[\partial_{k}+A_{k}, F_{k j}\right] .
$$

The Hamiltonian and the form are invariant with respect to the gauge transformations

$$
\mathbf{A} \rightarrow \mathbf{A}^{f}=f^{-1} d f+f^{-1} \mathbf{A} f, \mathbf{e} \rightarrow \mathbf{E}^{f}=f^{-1} \mathbf{E} f .
$$

where $d=\left(\partial_{1}, \partial_{2}, \partial_{3}\right)$. We assume that $f$ is a smooth map $f \in \mathcal{G}=C^{\infty}\left(\mathbb{R}^{3} \rightarrow G\right)$, vanishing at infinity and at some marked points

$$
f\left(\mathbf{y}^{a}\right)=0, \mathbf{y}^{a}=\left(y_{1}^{a}, y_{2}^{a}, y_{3}^{a}\right),(a=1, \ldots, n) .
$$

Infinitesimal gauge transformations defines the vector field on the phase space

$$
V_{\epsilon} \mathbf{E}=[\mathbf{E}, \epsilon], V_{\epsilon} \mathbf{A}=d \epsilon+[\mathbf{A}, \epsilon], \epsilon \in \operatorname{Lie}(\mathcal{G}) .
$$

The corresponding momentum Hamiltonian is

$$
F(\mathbf{E}, \mathbf{A}, \epsilon)=\int_{\mathbb{R} 3}\left\langle\epsilon\left(\sum_{j=1}^{3} \partial_{j} E_{j}+\left[A_{j}, E_{j}\right]\right)\right\rangle,
$$

(compare with (3.14)). Therefore the moment takes the form

$$
\mu(\mathbf{E}, \mathbf{A})=\sum_{j=1}^{3} \partial_{j} E_{j}+\left[A_{j}, E_{j}\right] .
$$

This is an element of the gauge co-algebra Lie* $(\mathcal{G})$. In other words the moment belongs to the map of the phase space $\mathcal{R}$ to the distributions on $\mathbb{R}^{3}$ with values in Lie $*(G)$.

Let us fix it as

$$
\sum_{j=1}^{3} \partial_{j} E_{j}+\left[A_{j}, E_{j}\right]=\sum_{a} \delta\left(\mathbf{x}-\mathbf{y}^{a}\right) \rho_{a},
$$

where $\rho_{a} \in$ Lie $^{*}(\mathcal{G})$. The moment constraints (3.21) are nothing else than the Gauss law, and $\rho_{a}$ are electric charges.

To come to the reduced phase space $\mathcal{R}^{\text {red }}=\mathcal{R} / / \mathcal{G}$ we should add a gauge fixing condition to the Gauss law. Note that the gauge transformations vanish at the points $\mathbf{y}^{a}$, and in this way they preserves the right hand side of (3.21). Starting with six fields $\left(A_{j}, E_{j}\right)$ defining $\mathcal{R}$, we put two types of constraints - the Gauss law and gauge fixing. Roughly speaking, they kill two fields and the reduced phase space describes the "transversal degrees of freedom".

\section{3d field theory}

\subsection{Hitchin systems}

\subsubsection{Fields}

Define a field theory on $(2+1)$ dimensional space-time of the form $\mathbb{R} \times \Sigma_{g, n}$, where is a Riemann surface of genus $g$ with a divisor $D=\left(x_{1}, \ldots, x_{n}\right)$ of $n$ marked points.

The phase space of the theory is defined by the following field content:

1) Consider a vector bundle $E$ of $\operatorname{rank} N$ over $\Sigma_{g, n}$ equipped with the connection $d^{\prime \prime}=\nabla_{\bar{z}} \otimes d \bar{z}$. It acts on the sections 


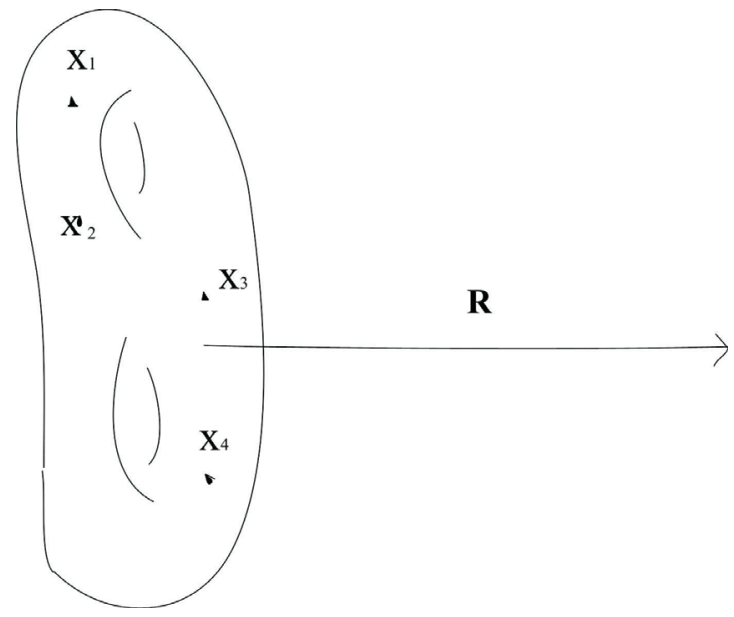

Fig. 1: $\left(x_{1}, \ldots, x_{4}\right)$-marked points

$s^{T}=\left(s_{1}, \ldots, s_{N}\right)$ of $E$ as $d^{\prime \prime} s=\bar{\partial} s+\bar{A} s$. The vector fields $\bar{A}(z, \bar{z})$ are $C^{\infty} \operatorname{maps} \Sigma_{g, n} \rightarrow \operatorname{gl}(N, \mathbb{C})$.

2) The scalar fields (the Higgs fields) $\Phi(z, \bar{z}) \otimes d z$, $\Phi: \Sigma_{g, n} \rightarrow \operatorname{gl}(N, \mathbb{C})$. The Higgs field is a section of the bundle $\Omega^{(1,0)}\left(\Sigma_{g, n}, \operatorname{End} E\right)$. This means that $\Phi$ acts on the sections $s_{j} \rightarrow \Phi_{k j} s_{j} \otimes d z$. We assume that $\Phi$ has holomorphic poles at the marked points $\Phi \sim \frac{\Phi^{a}}{z-x_{a}}+\ldots$

Let $\left(\alpha_{1}, \ldots, \alpha_{g}, \beta_{1}, \ldots, \beta_{g}\right)$ be a set of fundamental cycles of $\Sigma_{g, n},\left(\prod_{j} \alpha_{j} \beta_{j} \alpha_{j}^{-1} \beta_{j}^{-1}=1\right)$. The bundle $E$ is defined by the monodromy matrices $\left(Q_{j}, \Lambda_{j}\right)$

$$
\alpha_{j}: s \rightarrow Q_{j}^{-1} s, \beta_{j}: \Lambda_{j}^{-1} s
$$

Similarly, for $\bar{A}$ and $\Phi$ we have

$\alpha_{j}: \bar{A} \rightarrow Q_{j} \bar{\partial} Q_{j}^{-1}+Q_{j} \bar{A} Q_{j}^{-1}$,

$\beta_{j}: \bar{A} \rightarrow \Lambda_{j} \bar{\partial} \Lambda_{j}^{-1}+\Lambda_{j} \bar{A} \Lambda_{j}^{-1}$

$\alpha_{j}: \Phi \rightarrow Q_{j} \Phi Q_{j}^{-1}, b_{j}: \Phi \rightarrow \Lambda_{j} \Phi \Lambda_{j}^{-1}$.

3) The spin variables are attributed to the marked points $S^{a} \in \operatorname{gl}(N, \mathbb{C}), a=1, \ldots, n, S^{a}=g^{-1} S^{a}(0) g$, where $S^{a}(0)$ is a fixed element of $\operatorname{gl}(N, \mathbb{C})$. In other words, $S^{a}$ belong to coadjoint orbits $\mathcal{O}^{a}$ of $\operatorname{gl}(N, \mathbb{C})$. They play the role of non-abelian charges located at the marked points.

Let $\left\{T_{\alpha}\right\},\left(\alpha=1, \ldots, N^{2}\right)$ be a basis in the Lie algebra $\operatorname{gl}(N, \mathbb{C}),\left[T_{\alpha}, T_{\beta}\right]=C_{\alpha, \beta}^{\gamma} T_{\gamma}$. Define the Poisson structure on the space of fields:

1) Darboux brackets of the fields $(A, \Phi)$ :

$$
\begin{aligned}
& \bar{A}(z, \bar{z})=\sum_{\alpha} \bar{A}_{\alpha}(z, \bar{z}) T_{\alpha}, \Phi(w, \bar{w})=\sum_{\beta} \Phi_{\beta}(w, \bar{w}) T_{\beta} . \\
& \left\{\Phi_{\alpha}(w, \bar{w}), \bar{A}_{\beta}(z, \bar{z})\right\}=\left\langle T_{\alpha} T_{\beta}\right\rangle \delta(z-w, \bar{z}-\bar{w}), \\
& (\langle\rangle=\text { trace in ad })
\end{aligned}
$$

2) Linear Lie brackets for the spin variables:

$$
\begin{aligned}
& \mathbf{S}^{a}=\sum_{\alpha} S_{\alpha}^{a} T_{\alpha} \\
& \left\{S_{\alpha}^{a}, S_{\beta}^{b}\right\}=\delta^{a, b} C_{\alpha, \beta}^{\gamma} S_{\gamma}^{a} .
\end{aligned}
$$

In this way we have defined the phase space

$$
\mathcal{R}=\left(\bar{A}, \Phi, \mathbf{S}^{a}\right) \text {. }
$$

The Poisson brackets are non-degenerate and the space $\mathcal{R}$ is symplectic with the form

$$
\begin{aligned}
& \omega=\omega^{0}-\sum_{a=1}^{n} \int_{\Sigma_{g, n}} \omega^{a} \delta\left(z-x_{a}, \bar{z}-\bar{x}_{a}\right), \\
& \omega^{0}=\int_{\Sigma_{g, n}}\langle D \Phi \wedge D \bar{A}\rangle, \\
& \omega^{a}=\left\langle D\left(S^{a} g^{-1}\right) \wedge D g\right\rangle .
\end{aligned}
$$

The last form is the Kirillov-Kostant form on the coadjoint orbits. The fields $(\Phi, \bar{A})$ are holomorphic coordinates on $\mathcal{R}$ and the form $\omega^{0}$ is the (2,0)-form in this complex structure on $\mathcal{R}$. Similarly, $\left(S^{a} g^{-1}, g\right)$ are holomorphic coordinates on the orbit $\mathcal{O}^{a}$, and $\omega^{a}$ is also $(2,0)$ form.

\subsubsection{Hamiltonians}

The traces $\left\langle\Phi^{j}\right\rangle(j=1, \ldots, N)$ of the Higgs field are periodic $(j, 0)$-forms $\Omega^{(j, 0)}\left(\Sigma_{g, n}\right)$ with holomorphic poles of order $j$ at the marked points. To construct integrals from $\left\langle\Phi^{j}\right\rangle$ one should integrate them over $\Sigma_{g, n}$ and to this end prepare $(1,1)$-forms from the $(j, 0)$-forms. For this purpose consider the space of smooth $(1-j, 1)$-differentials $\Omega^{(1-j, 1)}\left(\Sigma_{g, n} \backslash D\right)$ vanishing at the marked points. Locally, they are represented as $\mu_{j}=\mu_{j}(z, \bar{z})\left(\frac{\partial}{\partial z}\right) j^{j-1} \otimes d \bar{z}$. In other words $\mu_{j}$ are $(0,1)$-forms taking values in degrees of vector fields $\mathcal{T}$ on $\Sigma_{g, n} \backslash D$. For example, $\mu_{2}$ is the Beltrami differential.

The product $\left\langle\Phi^{j}\right\rangle \mu_{j}$ can be integrated over the surface. We explain below that $\mu_{j}$ can be chosen as elements of the basis in the cohomology space $H^{1}\left(\Sigma_{g, n} \backslash D, \mathcal{T}^{\otimes j-1}\right)$. This space has dimension

$n_{j}=\operatorname{dim} H^{1}\left(\Sigma_{g, n} \backslash D, \mathcal{T}^{\otimes j-1}\right)=\left\{\begin{array}{cc}(2 j-1)(g-1)+j n & j>1 \\ g & j=1\end{array}(4.6)\right.$

Let $\mu_{j, k}$ be a basis in $H^{1}\left(\Sigma_{g, n} \backslash D, \mathcal{T}^{\otimes j-1}\right),\left(k=1, \ldots, n_{j}\right)$. The product $\mu_{j, k}\left\langle\Phi^{j}\right\rangle$ can be integrated to define the Hamiltonians

$$
I_{j, k}=\frac{1}{j} \int_{\Sigma_{g, n}} \mu_{j, k}\left\langle\Phi^{j}\right\rangle, J=1, \ldots, N .
$$

It follows from (4.6) that the number of independent integrals $\sum n_{j}$ for $\mathrm{GL}(N, \mathbb{C})$ is

$$
d_{N, g, n}=\sum_{j=1}^{N} n_{j}=(g-1) N^{2}+1+n \frac{N(N-1)}{2} .
$$

Since $\langle\Phi\rangle=0$ for $\operatorname{SL}(N, \mathbb{C})$ the number of independent integrals is

$$
d_{N, g, n}=\sum_{j=2}^{N} n_{j}=(g-1)\left(N^{2}-1\right)+n \frac{N(N-1)}{2} .
$$


The integrals $I_{(j, k)}$ are independent and Poisson commute

$$
\left\{I_{\left(j_{1}, k_{1}\right)}, I_{\left(j_{2}, k_{2}\right)}\right\}=0 \text {. }
$$

Thus we come to $d_{N, g, n}$ commuting flows on the phase space $\mathcal{R}\left(\bar{A}, \Phi, \mathbf{S}^{a}\right)$

$$
\begin{aligned}
& \frac{\partial}{\partial t_{j, k}} \Phi=\left\{\nabla I_{j, k}, \Phi\right\}=0, \\
& \frac{\partial}{\partial t_{j, k}} \bar{A}=\mu_{j, k} \Phi^{j-1}, \\
& \frac{\partial}{\partial t_{j, k}} \mathbf{S}^{a}=0 . \operatorname{Res} \Phi_{z=x_{a}}=S^{a}
\end{aligned}
$$

\subsubsection{Action and gauge symmetries}

The same theory can be described by the action

$$
\begin{aligned}
\mathcal{S}= & \sum_{j=2}^{N} \sum_{k=1}^{n_{i}} \int_{\mathbb{R}_{j, k}} \int_{\Sigma_{g, n}}\left(\left\langle\Phi \partial_{j, k} \bar{A}\right\rangle+\right. \\
& \left.\sum_{a=1}^{n} \delta\left(z-x_{a}, \bar{z}-\bar{x}_{a}\right)\left\langle\mathbf{S}^{a} g_{a}^{-1} \partial_{j, k} g_{a}\right\rangle-I_{j, k}\right) d t_{j, k}
\end{aligned}
$$

where the time-like Wilson lines at the marked points are included. group

The action is gauge invariant with respect to the gauge

$$
\mathcal{G}_{\mathbb{C}}=\left\{\text { smooth maps }: \Sigma_{g, n} \rightarrow \operatorname{GL}(N, \mathbb{C})\right\}
$$

The elements $f \in \mathcal{G}_{\mathbb{C}}$ are smooth and have the same monodromies as the Higgs field (4.1).

The action is invariant with respect to the gauge transformations

$$
\begin{aligned}
& \bar{A} \rightarrow f^{-1} \bar{\partial} f+f^{-1} \bar{A} f, \Phi \rightarrow f^{-1} \Phi f \\
& g_{a} \rightarrow g_{a} f^{a}, \mathbf{S}^{a} \rightarrow\left(f^{a}\right)^{-1} \mathbf{S}^{a} f^{a}, f^{a}=\left.f(z, \bar{z})\right|_{z=x_{a}} .
\end{aligned}
$$

Consider the infinitesimal gauge transformations $V_{\varepsilon} \bar{A}=\bar{\partial} \varepsilon+[\bar{A}, \varepsilon], V_{\varepsilon} \Phi=[\Phi, \varepsilon]$,

$V_{\varepsilon} g_{a}=g_{a} \varepsilon\left(x_{a}\right), V_{\varepsilon} S^{a}=\left[S^{a}, \varepsilon\left(x_{a}\right)\right], \varepsilon \in \operatorname{Lie}\left(\mathcal{G}_{\mathbb{C}}\right)$.

The Hamiltonian $F$ generating the gauge vector fields $\iota_{\varepsilon} \omega=D F$ has the form

$F=\int_{\Sigma_{g, n}}\left\langle\varepsilon\left(\bar{\partial} \Phi+[\bar{A}, \Phi]-\sum_{a=1}^{n} \mathbf{S}^{a} \delta\left(z-x_{a}, \bar{z}-\bar{x}_{a}\right)\right)\right\rangle$.

The moment map

$$
\begin{aligned}
& \mu: \mathcal{R}\left(\bar{A}, \Phi, \mathbf{S}^{a}\right) \rightarrow \text { Lie }^{*}(\mathcal{G C}), \\
& \mu=\bar{\partial} \Phi+[\bar{A}, \Phi]-\sum_{a=1}^{n} \mathbf{S}^{a} \delta\left(z-x_{a}, \bar{z}-\bar{x}_{a}\right) .
\end{aligned}
$$

The Gauss law (the moment constraints) takes the form

$$
\bar{\partial} \Phi+[\bar{A}, \Phi]=\sum_{a=1}^{n} \mathbf{S}^{a} \delta\left(z-x_{a}, \bar{z}-\bar{x}_{a}\right) .
$$

Upon imposing these constraints the residues of the Higgs fields become equal to the spin variables by analogy
$\operatorname{Res} \Phi_{z=x_{a}}=S^{a}$ with the Yang-Mills theory, where the Higgs field corresponds to the electric field and $\mathbf{S}^{a}$ are the analog of the electric charges.

The reduced phase space $\mathcal{R}^{\text {red }}=\mathcal{R}\left(\bar{A}, \Phi, \mathbf{S}^{a}\right) /($ Gauss law $)+$ (gauge fixing $)$

defines the physical degrees of freedom, and the reduced phase space is the symplectic quotient

$$
\mathcal{R}^{\text {red }}=\mathcal{R}\left(\bar{A}, \Phi, \mathbf{S}^{a}\right) / / \mathcal{G}_{\mathbb{C}}
$$

\subsubsection{Algebra-geometric approach}

The operator $d^{\prime \prime}$ acting on sections defines a holomorphic structure on the bundle $E$. A section $s$ is holomorphic if

$$
(\bar{\partial}+\bar{A}) s=0
$$

The moment constraint (4.14) means that the space of sections of the Higgs field over $\Sigma_{g} \backslash D$ is holomorphic.

Consider the set of holomorphic structures $\mathcal{L}=\left\{d_{\bar{A}}\right\}$ on $E$. Two holomorphic structure are called equivalent if the corresponding connections are gauge equivalent. The moduli space of holomorphic structures is the quotient $\mathcal{L} / \mathcal{G}_{\mathbb{C}}$. Generically the quotient has very singular structure. To have a reasonable topology one should consider the so-called stable bundles. The stable bundles are generic and we consider the space of connection $\mathcal{L}^{\text {stable }}$ corresponding to the stable bundles. The quotient is called the moduli space of stable holomorphic bundles

$$
\mathcal{M}(N, g, n)=\mathcal{L}^{\text {stable }} / \mathcal{G} .
$$

It is a finite-dimensional manifold. The tangent space to $\mathcal{M}(N, g, n)$ is isomorphic to $H^{1}\left(\Sigma_{g, n}, \operatorname{End} E\right)$. Its dimension can be extracted from the Riemann-Roch theorem and for curves without marked points $(n=0)$ $\operatorname{dim} H^{0}(\Sigma, \operatorname{End} E)-\operatorname{dim} H^{1}(\Sigma, \operatorname{End} E)=(1-g) \operatorname{dim} G$.

For stable bundles and $g>1$ and $\operatorname{dim} H^{0}(\Sigma$, End $E)=1$ and

$$
\operatorname{dim} \mathcal{M}(N, g, 0)=(g-1) N^{2}+1
$$

for $\operatorname{GL}(N, \mathbb{C})$, and

$$
\operatorname{dim} \mathcal{M}(N, g, 0)=(g-1)\left(N^{2}-1\right)
$$

for $\operatorname{SL}(N, \mathbb{C})$.

Thus, in the absence of the marked points we should consider bundles over curves of genus $g \geq 2$. But the curves of genus $g=0$ and 1 are important for applications to integrable systems. Including the marked points improves the situation.

We extend the moduli space by providing an additional data at the marked points. Consider an $\mathrm{N}$-dimensional vector space $V$ and choose a flag $F l=\left(V_{1} \subset V_{2} \subset \ldots V_{N}=V\right)$, where $V_{j}$ is a subspace in $V_{j+1}$. Note that a flag is a point in a homogeneous space called the flag variety $F l \in \mathrm{GL}(N, \mathbb{C}) / B$, where $B$ is a Borel subgroup. If $\left(e_{1}, \ldots, e_{N}\right)$ is a basis in $V$ and $F l$ is a flag $F l=\left\{V_{1}=\left\{a_{11} e_{1}\right\}, V_{2}=\left\{a_{21} e_{1}+a_{22} e_{2}\right\}, \ldots V_{N}=V\right\}$, 
then $B$ is the subgroup of lower triangular matrices. The flag variety has the dimension $\frac{1}{2} N(N-1)$. The moduli space $\mathcal{M}(N, g, n)$ is the moduli space $\mathcal{M}(N, g, 0)$ equipped with maps $g_{a} \in \mathrm{GL}(N, \mathbb{C})$ of $V$ to the fibers over the marked points $\left.V \rightarrow E\right|_{x_{a}}$, preserving $F l$ in $V$. In other words $g_{a}$ are defined up to the right multiplication of $B$ and therefore we supply the moduli space $\mathcal{M}(N, g, 0)$ with the structure of the flag variety $\operatorname{GL}(N, \mathbb{C}) / B$ at the marked points. We have a natural "forgetting" projection $\pi: \mathcal{M}(N, g, n) \rightarrow \mathcal{M}(N, g, 0)$. The fiber of this projection is the product of copies of the flag varieties. Bundles with this structure are called the quasi-parabolic bundles. The dimension of the moduli space of quasi-parabolic holomorphic bundles is

$$
\operatorname{dim} \mathcal{M}(N, g, n)=\mathcal{M}(N, g, 0)+\frac{1}{2} n N(N-1) .
$$

For curves of genus $g>1, \operatorname{dim} \mathcal{M}(N, g, n)$ is independent on the degree of the bundles $d=\operatorname{deg}(E)=c_{1}(\operatorname{det} E)$. In fact, we have a disjoint union of components labeled by the corresponding degrees of the bundles $\mathcal{M}=\coprod \mathcal{M}^{(d)}$. For elliptic curves $(g=1)$ one has

$$
\operatorname{dim} H^{1}(\Sigma, \operatorname{End} E)=\operatorname{dim} H^{0}(\Sigma, \operatorname{End} E),
$$

and $\operatorname{dim} H^{0}(\Sigma, \operatorname{End} E)$ does depend on $\operatorname{deg}(E)$. Namely,

$$
\operatorname{dim}(\mathcal{M}(N, 1,0, d))=\text { g.c.d. }(N, d) .
$$

In this case the structure of the moduli space for trivial bundles (i.e. with $\operatorname{deg}(E)=0$ ) and, for example, for bundles with $\operatorname{deg}(E)=1$ is different.

Now consider the Higgs field $\Phi$. As we already mentioned, $\Phi$ defines an endomorphism of the bundle $E$

$$
\Phi: \Omega^{(0)}\left(\Sigma_{g, n}, E\right) \rightarrow \Omega^{(1,0)}\left(\Sigma_{g, n}, E\right), s \rightarrow \Phi s \otimes d z .
$$

Similarly, they can be described as sections of $\Omega_{C^{\infty}}^{(0)}\left(\Sigma_{g, n}, \operatorname{End} E \otimes K_{D}\right)$. Here $K_{D}$ is the canonical class on $\Sigma \backslash D$ that locally apart from $D$ is represented as $d z$. Rememeber that $\Phi$ has poles at $D$. On the other hand, as it follows from the definition of the symplectic structure (4.4) on the set of pairs $(\Phi, \bar{A})$, that the Higgs field plays the role of a "covector" with respect to vector $\bar{A}$. In this way the Higgs field $\Phi$ is a section of the cotangent bundle $T^{*} \mathcal{L}^{\text {stable }}$.

The pair of the holomorphic vector bundle and the Higgs field $(E, \Phi)$ is called the Higgs bundle. The reduced phase space (4.15) is the moduli space of the quasi-parabolic Higgs bundles. It is the cotangent bundle

$$
\mathcal{R}^{\text {red }}=T^{*} \mathcal{M}(N, g, n, d) \text {. }
$$

Due to the Gauss law (4.14), the Higgs fields are holomorphic on $\Sigma \backslash D$. Then on the reduced space $\mathcal{R}^{\text {red }}$

$$
\Phi \in H^{0}\left(\Sigma_{g, n}, \text { End }^{*} E \otimes K_{D}\right) .
$$

A part of $T^{*} \mathcal{M}(N, g, n, d)$ comes from the cotangent bundle to the flag varieties $T^{*}(G / B)_{a}$ located at the marked points. Without the null section $T^{*}(G / B)_{a}$ is isomorphic to a unipotent coadjoint orbit, while the null section is the trivial orbit. Generic coadjoint orbits passing through a semi-simple element of $\operatorname{gl}(N, \mathbb{C})$ are affine spaces over $T^{*}(G / B)_{a}$. In this way we come to the moduli space of the quasi-parabolic Higgs bundles [29]. It has dimension

$\operatorname{dim} \mathcal{R}^{\text {red }}=2 N^{2}(g-1)+2+N(N-1) n$

This formula is universal and valid also for $g=0,1$ and does not depend on $\operatorname{deg}(E)$. At first glance, for $g=1$ this formula appears to contradict to (4.16). In fact, we have a residual gauge symmetry generated by a subgroup of the Cartan group of $\mathrm{GL}(N, \mathbb{C})$. The symplectic reduction with respect to this symmetry kill these degrees of freedom and we come to $\operatorname{dim} \mathcal{R}^{\text {red }}=2+N(N-1) n$ (see (4.6). We explain this mechanism on a particular example in Section 4.2.2. Formula (4.6) suggests that the phase spaces corresponding to bundles of different degrees may be symplectomorphic. We will see soon this is the case.

It follows from (4.18) that $\left\langle\Phi^{j}\right\rangle \in H^{0}\left(\Sigma_{g, n}, K_{D}^{j}\right)$. In other words $\left\langle\Phi^{j}\right\rangle$ are meromorphic forms on the curve with poles of the order $j$ at the divisor $D$. Let $\xi^{j k}$ be a basis of $H^{0}\left(\Sigma_{g, n}, K_{D}^{j}\right)$. Then

$$
\frac{1}{j}\left\langle\Phi^{j}\right\rangle=\sum_{k=1}^{n_{i}} I_{j k} \xi^{j k} .
$$

The basis $\mu_{j k}$ in $H^{1}\left(\Sigma_{g, n} \backslash D, \mathcal{T}^{\otimes j-1}\right)$ introduced above is dual to the basis $\varepsilon^{j k}$

$$
\int_{\Sigma_{g, n}} \mu_{j k} \xi^{l m}=\delta_{j}^{l} \delta_{k}^{m}
$$

Then the coefficients of the expansion (4.20) coincide with the integrals (4.7). The dimensions $n_{j}$ (4.6) can be calculated as $\operatorname{dim} H^{0}\left(\Sigma_{g, n}, K_{D}^{j}\right)$.

Symplectic reduction preserves the involutivity (4.10) of the integrals (4.7). Since

$$
\frac{1}{2} \operatorname{dim} \mathcal{T}^{*} \mathcal{M}(N, g, n)=\text { number of integrals }
$$

(see(4.8), (4.9)) we come to integrable systems on the moduli space of the quasi-parabolic Higgs bundles $\mathcal{R}^{\text {red }}$.

For $\operatorname{GL}(N, \mathbb{C})$ the Liouville torus is the Jacobian of the spectral curve $\mathcal{C}(2.5)$. Consider bundles with the structure group replaced by a reductive group $G$. The algebraic integrability for $g>1$ and $G$ is a classical simple group was proved in [1]. The case of exceptional groups was considered in [30, $31]$.

\subsubsection{Equations of motion on the reduced phase space}

Let us fix a gauge $\bar{A}=\bar{A}_{0}$. For an arbitrary connection $\bar{A}$ define a gauge transform 
$f[\bar{A}]: \bar{A} \rightarrow \bar{A}_{0}, \bar{A}_{0}=\left(f^{-1} \bar{\partial} f\right)[\bar{A}]+f^{-1}[\bar{A}] \bar{A} f[\bar{A}]$

Then $f[\bar{A}]$ is an element of the coset space $\mathcal{G} \mathbb{C} / \mathcal{G}_{0}$, where the subgroup $\mathcal{G}_{0}$ preserves the gauge fixing

$$
\mathcal{G}_{0}=\left\{f \mid \bar{\partial} f+\left[\bar{A}_{0}, f\right]=0\right\} .
$$

The same gauge transformation brings the Higgs field to the form

$$
L=f^{-1}[\bar{A}] \Phi f[\bar{A}] .
$$

The equations of motion for $\Phi$ (4.11) in terms of $L$ take the form of the Lax equation

$$
\partial_{j, k} L=\left[L, M_{j, k}\right]
$$

where $M_{j, k}=f^{-1}[\bar{A}] \partial_{j, k} f[\bar{A}]$. Therefore, after reduction the Higgs field becomes the Lax matrix. Equations (4.21) describes the Hitchin integrable hierarchy.

The matrix $M_{j, k}$ can be extracted from the second equation (4.12)

$$
\bar{\partial} M_{j, k}-\left[M_{j, k}, \bar{A}_{0}\right]=\partial_{j, k} \bar{A}_{0}-L^{j-1} \mu_{j, k} .
$$

The Gauss law restricted to $\mathcal{R}^{\text {red }}$ takes the form

$$
\bar{\partial} L+\left[\bar{A}_{0}, L\right]=\sum_{a=1}^{n} \mathbf{S}^{a} \delta\left(x_{a}, \bar{x}_{a}\right)
$$

Thus, the Lax matrix is the matrix Green function of the operator $\bar{\partial}+\bar{A}_{0}$ on $\Sigma_{g, n}$ acting in the space $\Omega^{(1,0)}\left(\Sigma_{g, n}, \operatorname{End} E\right)$.

The linear system corresponding to the integrable hierarchy takes the following form. Consider a section $\psi$ of the vector bundle $E$. The section is called the Baiker-Akhiezer function if it is a solution of the linear system for

$$
\begin{cases}\text { 1. } & \left(\bar{\partial}+\bar{A}_{0}\right) \psi=0, \\ \text { 2. } & (\lambda-L) \psi=0, \\ \text { 3. } & \left(\partial_{j, k}+M_{j, k}\right) \psi=0 .\end{cases}
$$

The first equation means that $\psi$ is a holomorphic section. Compatibility of the first equation and the second equation is the Gauss law (4.23) and the first equation and the last equation is the Lax equations (4.21).

In terms of the Lax matrix the integrals of motion $I_{j, k}$ are expressed by the integrals (4.7)

$$
I_{j k}=\frac{1}{j} \int_{\Sigma g, n} \mu_{j k} \operatorname{tr}(L(x, z))^{j}
$$

or by the expansion (4.20)

$$
\frac{1}{j}\left\langle L^{j}\right\rangle=\sum_{k=1}^{n_{i}} I_{j k} \xi^{j k}
$$

The moduli space of the Higgs bundles (4.17) is parameterized by the pairs $\left(\mathbf{A}_{0}, L\right)$. The projection (2.2)

$$
T^{*} \mathcal{M}(N, g, n) \rightarrow B=\sum_{j=1}^{N} H^{0}\left(\Sigma_{g, n} \backslash D, K_{D}^{j}\right)
$$

is called the Hitchin fibration.

An illustrative example of the Hitchin construction is the Higgs bundles over elliptic curves. These cases will be described explicitly in following subsections.

\subsection{N-body Elliptic Calogero-Moser System (ECMS)}

\subsubsection{Description of the system}

Let $C \tau$ be an elliptic curve $\mathbb{C} /(\mathbb{Z}+\tau \mathbb{Z}), \operatorname{Im} \tau>0$. The phase space $\mathcal{R}^{E C M}$ of ECMS is described by $N$ complex coordinates and their momenta

$\begin{cases}\mathbf{u}=\left(u_{1}, \ldots, u_{N}\right) & \left(u_{j} \in C_{\tau}\right)-\text { coordinates of particles }, \\ \mathbf{v}=\left(v_{1}, \ldots, v_{N}\right) & \left(u_{j} \in \mathbb{C}\right)-\text { momentum vector }\end{cases}$

with the Poisson brackets $\left\{v_{j}, u_{k}\right\}=\delta_{j k}$.

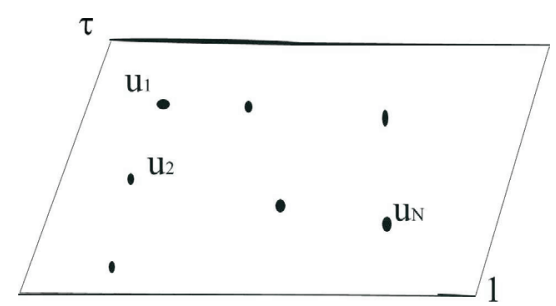

Fig. 2

The Hamiltonian takes the form

$$
H^{C M}=\frac{1}{2}|\mathbf{v}|^{2}+v^{2} \sum_{j<k} \wp\left(u_{j}-u_{k}\right) .
$$

Here $v^{2}$ is a coupling constant and $\wp(z)-$ is the Weierschtrass function. It is a double periodic meromorphic function $\wp(z+1)=\wp(z+\tau) \wp(z)$ with a second order pole $\wp(z) \sim z^{-2}$, $z \rightarrow 0$.

The system has the Lax representation [39] with the Lax matrix

$$
\begin{array}{r}
L^{C M}=i V+X, V=\operatorname{diag}\left(v_{1}, \ldots, v_{N}\right), \\
X_{j k}=v \mathbf{e}\left(\frac{z-\bar{z}}{\tau-\bar{\tau}}\left(u_{j}-u_{k}\right)\right) \phi\left(u_{j}-u_{k}, z\right), \\
\mathbf{e}(x)=\exp 2 \pi i x
\end{array}
$$

where

$$
\phi(u, z)=\frac{\theta(u+z) \theta^{\prime}(0)}{\theta(u) \theta(z)}
$$

and

$\theta(z)=q^{\frac{1}{8}} \sum_{n \in \mathbf{Z}}(-1)^{n} \exp 2 \pi \iota\left(\frac{1}{2} n(n+1) \tau+n z\right)$,

$q=\exp 2 \pi i \tau$

is the standard theta-function with a simple zero at $z=0$ and the monodromies

$\theta(z+1)=-\theta(z), \theta(z+\tau)=-q^{-\frac{1}{2}} e^{-2 \pi i z} \theta(z)$.

Then from (4.31) that

$\phi(u, z+1)=\phi(u, z), \phi(u, z+\tau)=\mathbf{e}(-u) \phi(u, z)$,

and $\phi(u, z)$ has a simple pole at $z=0$

$$
\left.\operatorname{Res} \phi(u, z)\right|_{z=0}=1
$$




\subsubsection{ECMS and Higgs bundles [6, 7]}

To describe ECMS as a Hitchin system consider a vector bundle $E$ of rank $N$ and degree 0 over an elliptic curve $\Sigma_{1,1}$ with one marked point. We assume that the curve is isomorphic to $C_{\tau}=\mathbb{C} /(\mathbb{Z}+\tau \mathbb{Z})$. The quasi-parabolic Higgs bundle $T^{*} E$ has coordinates $\mathcal{R}^{0}=\{\Phi(z, \bar{z}), \bar{A}(z, \bar{z}), S\}, \quad \Phi, \bar{A} \in \operatorname{gl}(N, \mathbb{C}), \quad S \in \mathcal{O}$, where $\mathcal{O}$ is a degenerate orbit at the marked point $z=0$

$$
\mathcal{O}=\left\{S=g^{-1} S^{0} g \mid g \in \operatorname{GL}(N, \mathbb{C}), S^{0}=v J\right\},
$$

and $J$ is the matrix (3.17). The orbit has dimension

$$
\operatorname{dim}(\mathcal{O})=2 N-2 \text {. }
$$

For degree zero bundles the monodromies around the two fundamental cycles can be chosen as $Q_{1}=I_{d}$ and $\Lambda_{1}=\mathbf{e}(\mathbf{u})$, where $\mathbf{e}(\mathbf{u})=\operatorname{diag}\left(\left(\exp 2 \pi i u_{1}, \ldots, \exp 2 \pi i u_{N}\right)\right.$. A section with these monodromies is

$$
s^{T}=\left(s_{1}, \ldots, s_{N}\right), s_{j}=\phi\left(u_{j}, z\right) .
$$

where $\phi\left(u_{j}, z\right)$ is (4.29). It follows from (4.32) that the section has the prescribed monodromies.

For the fields and the gauge group we have the same monodromies

$$
\bar{A}(z+1)=\bar{A}(z), \Phi(z+1)=\Phi(z),
$$

$\bar{A}(z+\tau)=\mathbf{e}(\mathbf{u}) \bar{A}(z) \mathbf{e}(-\mathbf{u}), \Phi(z+\tau)=\mathbf{e}(\mathbf{u}) \Phi(z) \mathbf{e}(-\mathbf{u})$,

$f(z+1, \bar{z}+1)=f(z, \bar{z}), f(z+\tau, \bar{z}+\bar{\tau})=\mathbf{e}(\mathbf{u}) f(z, \bar{z}) \mathbf{e}(-\mathbf{u})$.

It can be proved that for bundles of degree zero generic connections $\bar{A}=-\bar{\partial} f f^{-1}$ is trivial and therefore

$$
\bar{A} \rightarrow \bar{A}_{0}=0 \text {. }
$$

This means that stable bundles $E$ of rank $N$ are decomposed into the direct sum of line bundles

$$
E=\oplus_{j=1}^{N} \mathcal{L}_{j},
$$

with the sections (4.34). The elements $u_{j}$ are the points of the Jacobian $\operatorname{Jac}\left(\Sigma_{\tau}\right)$. They play the role of coordinates, and thereby, $C_{\tau} \sim \operatorname{Jac}\left(\Sigma_{\tau}\right)$.

This gauge fixing is invariant with respect to the constant diagonal subgroup $D_{0}$. It acts on the spin variables $S \in \mathcal{O}$. This action is Hamiltonian. The moment equation of this action is $\operatorname{diag}(\mathcal{O})=0$. This condition dictates the form of $S^{0}=J$. Gauge fixing allows one to kill the degrees of freedom related to the spin variables, because $\operatorname{dim}(\mathcal{O})=2(N-1)$ and $\operatorname{dim}\left(D_{0}\right)=N-1$. Thus, the symplectic quotient is a point $\left(\operatorname{dim}\left(\mathcal{O} / / D_{0}\right)=0\right)$.

Remark 4.1 One can choose an arbitrary orbit $\mathcal{O}$. In this case we come to the symplectic quotient $\mathcal{O} / / D_{0}$. It has dimension $\operatorname{dim}(\mathcal{O})=2(N-1)$.

Now consider solutions the moment equation (4.23) with the prescribed monodromies and prove that $\Phi$ becomes the
Lax matrix $\Phi \rightarrow L^{C M}=V+X$ (4.27). Since $\bar{A}_{0}=0, V$ does not contribute in (4.23) and its elements are free parameters. We identify them with momenta of the particles $V=\operatorname{diag}\left(v_{1}, \ldots, v_{N}\right)$. Due to the term with the delta-function in (4.23) the off-diagonal part should have a simple pole with the residue $v J$ and the prescribed monodromies. It follows from (4.32) and (4.33) that $X_{j k}$ satisfies these conditions. They uniquely fix its matrix elements.

The reduced space is described by the variables $\mathbf{v}$ and $\mathbf{u}$. The symplectic form on the reduced space

$$
\int_{\Sigma_{1,1}}\left\langle L^{C M}, \bar{A}_{0}\right\rangle=\sum D v_{j} \wedge D u_{j}
$$

leads to the brackets $\left\{v_{j}, u_{k}\right\}=\delta_{j k}$.

From the general construction the integrals of motion come from the expansion of $\operatorname{tr}\left(L^{C M}\right)^{j}(\mathbf{v}, \mathbf{u}, z)$. They are double periodic meromorphic functions with poles at $z=0$. This is a finite-dimensional space generated by a basis of derivative of the Weierschtrass functions. They are elements of the basis $\xi^{j k}$ in (4.25).

$\frac{1}{j} \operatorname{tr}\left(L^{C M}\right)^{j}(\mathbf{v}, \mathbf{u}, z)=I_{0 j}^{C M}+I_{2 j}^{C M} \wp(z)+\ldots+I_{j j}^{C M} \wp^{(j)}(z)$.

There are $\frac{N(N+1)}{2}-1$ integrals. Due to a special choice of the orbit only $N-1$ integrals are independent. In particular,

$$
\frac{1}{2} \operatorname{tr}\left(L^{C M}\right)^{2}(\mathbf{v}, \mathbf{u}, z)=-H^{C M}+v^{2} \wp(z) .
$$

For generic orbits (see Remark 4.1) the Hamiltonian take the form

$$
H^{C M}=\frac{1}{2}|\mathbf{v}|^{2}+\sum_{j<k} S_{j k} S_{k j} \wp\left(u_{j}-u_{k}\right) .
$$

This is ECMS with spin [34]. Note, that $I_{j, j}$ are the Casimir functions defining a generic orbit $\mathcal{O}$. Therefore we have $\frac{N(N+1)}{2}-1-(N-1)=\frac{N(N-1)}{2}$ commuting integrals of motion. The number of independent commuting integrals is always equal to $\frac{1}{2} \operatorname{dim}(\mathcal{O})$.

\subsection{Elliptic Top $($ ET) on $\mathrm{GL}(N, \mathbb{C})$}

\subsubsection{Description of the system}

The elliptic top is an example of Euler-Arnold top related to the group $\mathrm{GL}(N, \mathbb{C})$. Its phase space is a coadjoint orbit of $\operatorname{GL}(N, \mathbb{C})$. The Hamiltonian is a quadratic form on the coalgebra $\mathfrak{g}^{*}=\operatorname{gl}(N, \mathbb{C})^{*}$. The ET is an integrable Euler-Arnold top. Before defining the Hamiltonian introduce a special basis in the Lie algebra $\operatorname{gl}(N, \mathbb{C})$. Define the finite set $\mathbb{Z}_{N}^{(2)}=(\mathbb{Z} / N \mathbb{Z} \oplus \mathbb{Z} / N \mathbb{Z}), \widetilde{\mathbb{Z}}_{N}^{(2)}=(\mathbb{Z} / N \mathbb{Z} \oplus \mathbb{Z} / N \mathbb{Z}) \backslash(0,0)$ and let $\mathbf{e}_{N}(x)=\exp \frac{2 \pi i}{N} x$. Then a basis is generated by $N^{2}-1$ matrices 


$$
T_{\alpha}=\frac{N}{2 \pi i} \mathbf{e}_{N}\left(\frac{\alpha_{1} \alpha_{2}}{2}\right) Q^{\alpha_{1}} \Lambda^{\alpha_{2}}, \alpha=\left(\alpha_{1}, \alpha_{2}\right) \in \widetilde{\mathbb{Z}}_{N}^{(2)},
$$

where

$$
\begin{aligned}
Q & =\operatorname{diag}\left(1, \mathbf{e}_{N}(1), \ldots, \mathbf{e}_{N}(N-1)\right), \\
\Lambda & =\sum_{j=1, N,(\bmod N)} E_{j, j+1}
\end{aligned}
$$

The commutation relations in this basis have a simple form

$$
\left[T_{\alpha}, T_{\beta}\right]=\frac{N}{\pi} \sin \frac{\pi}{N}(\alpha \times \beta) T_{\alpha+\beta} .
$$

Let $\mathbf{S}=\sum_{\alpha \in \mathbb{Z}_{N}^{(2)} \backslash(0,0)} S_{\alpha} T_{\alpha} \in \mathfrak{g}^{*}$. The Poisson brackets for the linear functions $S_{\alpha}$ come from the Lie brackets

$$
\left\{S_{\alpha}, S_{\beta}\right\}=\frac{N}{\pi} \sin \frac{\pi}{N}(\alpha \times \beta) S_{\alpha+\beta} .
$$

The phase space $\mathcal{R}^{E T}$ of the ET is a coadjoint orbit $\mathcal{R}^{E T} \sim \mathcal{O}=\left\{\mathbf{S} \in \mathfrak{g}^{*} \mid \mathbf{S}=g \mathbf{S}_{0} g^{-1}, g \in \mathrm{GL}(N, \mathbb{C})\right\}$.

A particular orbit passes through $\mathbf{S}_{0}=v J$, as for the spinless ECMS

The Euler-Arnold Hamiltonian is defined by the quadratic form

$$
H^{E T}=-\frac{1}{2} \operatorname{tr}(\mathbf{S} \cdot \mathbf{J}(\mathbf{S})),
$$

where $\mathbf{J}$ is diagonal in the basis $T_{\alpha}$

$$
\mathbf{J}(\mathbf{S}): S_{\alpha} \rightarrow \wp_{\alpha} S_{\alpha}, \wp_{\alpha}=\wp\left(\frac{\alpha_{1}+\alpha_{2} \tau}{N}\right), \alpha \in \overline{\mathbb{Z}}_{N}^{(2)} \text {. }
$$

The equations of motion corresponding to this Hamiltonian take the form

$$
\begin{aligned}
& \partial_{t} \mathbf{S}=\left\{H^{E T}, \mathbf{S}\right\}=[\mathbf{J}(\mathbf{S}), \mathbf{S}], \\
& \partial_{t} S_{\alpha}=\frac{N}{\pi} \sum_{\gamma \in \widetilde{\mathbb{Z}}_{N}^{(2)}} S_{\gamma} S_{\alpha-\gamma} \wp_{\gamma} \sin \frac{\pi}{N}(\alpha \times \gamma) .
\end{aligned}
$$

\subsubsection{Field theory and Higgs bundles}

The curve $\Sigma_{1,1}$ is the same as for the Calogero-Moser system. Consider a vector bundle $E$ of a rank $N$ and degree one over $\Sigma_{1,1}$. It is described by its sections $s=\left(s_{1}(z, \bar{z}), \ldots, s_{N}(z, \bar{z})\right)$ with monodromies

$$
\begin{gathered}
s^{T}(z+1, \bar{z}+1)=Q^{-1} s^{T}(z, \bar{z}), \\
s^{T}(z+\tau, \bar{z}+\bar{\tau})=\widetilde{\Lambda}^{-1} s^{T}(z, \bar{z}),
\end{gathered}
$$

where $Q$ is (4.37), $\widetilde{\Lambda}=\mathbf{e}_{N}^{-\left(z+\frac{\tau}{2}\right)} \Lambda$, and $\Lambda$ is (4.38). Since $\operatorname{det} Q= \pm 1$ and $\operatorname{det} \widetilde{\Lambda}= \pm \mathbf{e}_{1}^{-\left(z+\frac{\tau}{2}\right)}$ the determinants of the transition matrices have the same quasi-periods as the Jacobi theta-functions. The theta-functions have a simple pole on $\Sigma_{1,1}$. Thereby, the vector bundle $E_{N}$ has degree one.

The Higgs bundle has the same field content as ECMS

The orbit

$$
\mathcal{R}=\{\bar{A}, \Phi, \mathbf{S}\}, \bar{A}, \Phi \in \operatorname{gl}(N, \mathbb{C}), \mathbf{S} \in \mathcal{O} .
$$

$$
\mathcal{O}=\left\{\mathbf{S}=g^{-1} \mathbf{S}^{0} g, g \in \mathrm{GL}(N, \mathbb{C})\right\}
$$

is located at the marked point $z=0$.
It follows from (4.39) that the fields $\Phi, \bar{A}$ have the monodromies

$$
\begin{aligned}
& \bar{A}(z+1)=Q \bar{A}(z) Q^{-1}, \Phi(z+1)=Q \Phi(z) Q^{-1} \\
& \bar{A}(z+\tau)=\Lambda \bar{A}(z) \Lambda^{-1}, \Phi(z+\tau)=\Lambda \Phi(z) \Lambda^{-1}
\end{aligned}
$$

The group of the automorphisms $\mathcal{G}_{\mathbb{C}}=\{f\}$ of $E$ should have the same monodromies

$$
f(z+1)=Q f(z) Q^{-1}, f(z+\tau)=\Lambda f(z) \Lambda^{-1} .
$$

Due to the monodromy conditions the generic field $\bar{A}$ is gauge equivalent to the trivial $f^{-1} \bar{A} f+f^{-1} \bar{\partial} f=0$. Therefore

$$
\bar{A}=-\bar{\partial} f[\bar{A}] f^{-1}[\bar{A}] .
$$

This allows us to choose $\bar{A}=0$ as an appropriate gauge. It means that there are no moduli of holomorphic vector bundles. More precisely, the holomorphic moduli are related only to the quasi-parabolic structure of $E$ related to the spin variables $\mathbf{S}$. The monodromies of the gauge matrices prevent the existence of nontrivial residual gauge symmetries. Let $f[\bar{A}](z, \bar{z})$ be a solution of (4.40). Consider the transformation of $\Phi$ by solutions of (4.40)

$$
L^{E T}[\bar{A}, g](z, \bar{z})=f[\bar{A}](z, \bar{z}) \Phi f^{-1}[\bar{A}](z, \bar{z})
$$

The moment constraints (4.14) take the form

$$
\bar{\partial} L^{E T}=\delta(z, \bar{z}) \mathbf{S}
$$

The solution takes the form

$$
L^{E T}=\sum_{\alpha \in \mathbb{Z}_{N}^{(2)} \backslash(0,0)} S_{\alpha} \varphi_{\alpha}(z) T_{\alpha},
$$

where $\varphi_{\alpha}(z)=\mathbf{e}_{N}\left(\alpha_{2} z\right) \phi\left(\frac{\alpha_{1}+\alpha_{2} t}{N}, z\right)$. The Lax matrix was found in [40] using another approach. It is the Lax matrix of the vertex spinchain. The Lax matrix is meromorphic on $\Sigma_{1,1}$ with a simple pole with $\left.\operatorname{Res} L^{E T}\right|_{z=0}=\mathbf{S}$. The monodromies of $\varphi_{\alpha}(\mathrm{z})$ are read off from (4.32)

$\varphi_{\alpha}(z+1)=\mathbf{e}_{N}\left(\alpha_{2}\right) \varphi_{\alpha}(z), \varphi_{\alpha}(z+\tau)=\mathbf{e}_{N}\left(-\alpha_{1}\right) \varphi_{\alpha}(z)$

Then $L^{E T}$ have the prescribed monodromies. The reduced phase space $\mathcal{R}^{E T}$ is the coadjoint orbit:

$$
\mathcal{R}^{E T}=\left\{\mathcal{O}=\mathbf{S}=g \mathbf{S}_{0} g^{-1}\right\},
$$

$\mathbf{S}=\sum_{\alpha \in \mathbb{Z}_{N}^{(2)} \backslash(0,0)} S_{\alpha} T_{\alpha} \in \mathfrak{g}^{*}$. The symplectic form on $\mathcal{R}^{E T}$ is the Kirillov-Kostant form (4.5).

For a particular choice of the orbit passing through $J$ (refJ) its dimension coincide with the dimension of the phase of the spinless ECMS

$$
\operatorname{dim} \mathcal{R}^{E T}=\operatorname{dim} \mathcal{R}^{C M S}=2 N-2 .
$$

This is not occasional and we prove below that $\mathcal{R}^{C M}$ is symplectomorphic to $\mathcal{R}^{E T}$.

Since the traces $\operatorname{tr}\left(L^{E T}\right)^{j}$ are double periodic and have poles at $z=0$ the integrals of motion come from the expansion (see (4.36)) 


$$
\operatorname{tr}\left(L^{E T}(z)\right)^{k}=I_{0, k}+I_{2, k} \wp(z)+\ldots+I_{k, k} \wp^{(k-2)}(z)
$$

In particular,

$$
\operatorname{tr}\left(L^{E T}\right)^{2}=H^{E T}+C^{2} \wp(z)
$$

The coefficients $I_{s, k}$ are in involution

$$
\left\{I_{s, k}, I_{m, j}\right\}=0 \text {. }
$$

In particular, all functions $I_{s, k}$ Poisson commute with the Hamiltonian $H^{E T}$. Therefore, they play the role of conservation laws of elliptic rotator hierarchy on $\operatorname{GL}(N, \mathbb{C})$. We have a tower of $\frac{N(N+1)}{2}$ independent integrals of motion

$$
\begin{array}{ccccc}
I_{0,2} & I_{2,2} & & & \\
I_{0,3} & I_{2,3} & I_{3,3} & & \\
\ldots & \ldots & \ldots & \ldots & \\
I_{0, n} & I_{2, N} & \ldots & \ldots & I_{N, N}
\end{array}
$$

There are no integrals $I_{1, k}$ because there are no double periodic meromorphic functions with one simple pole. The integrals $I_{k, k}, k=0,2,3, \ldots, N$ are the Casimir functions corresponding to the coadjoint orbit

$$
\mathcal{R}^{E T}=\left\{S \in \operatorname{gl}(N, \mathbb{C}), \mathbf{S}=g^{-1} \mathbf{S}^{(0)} g\right\} .
$$

The conservation laws $I_{s, k}$ generate commuting flows on $\mathcal{R}^{\text {rot }}$

$$
\partial_{s, k} \mathbf{S}=\left\{I_{s, k}, \mathbf{S}\right\}_{1},\left(\partial_{s, k}:=\partial_{t_{s, k}}\right) .
$$

\subsection{Symplectic Hecke correspondence}

Let $E$ and $\widetilde{E}$ be two bundles over $\Sigma$ of the same rank. Assume that there is a map $\Xi^{+}: E \rightarrow \widetilde{E}$ (more precisely a map of the space of sections $\Gamma(E) \rightarrow \Gamma(\widetilde{E}))$ such that it is an isomorphism on the complement to $z_{0}$ and it has one-dimensional cokernel at $x \in \Sigma$ :

$$
\left.0 \rightarrow E \stackrel{\Xi^{+}}{\longrightarrow} \widetilde{E} \rightarrow C\right|_{z_{0}} \rightarrow 0
$$

The map $\Xi^{+}$is called upper modification of the bundle $E$ at the point $z_{0}$. Let $w=z-z_{0}$ be a local coordinate in a neighborhood of $z_{0}$. We represent locally $E$ as a sum of line bundles $E=\oplus_{j=1}^{N} \mathcal{L}_{j}$ with holomorphic sections

$$
s=\left(s_{1}, s_{2}, \ldots, s_{N}\right) .
$$

After modification we come to the bundle

$$
\widetilde{E}=\oplus_{j=1}^{N} \mathcal{L}_{j} \otimes \mathcal{O}\left(z_{0}\right)
$$

The sections of $\widetilde{E}$ are represented locally as $\widetilde{s}=\left(g_{1}(w) s_{1}, \ldots, w^{-1} g_{N}(w) s_{N}\right)$, where $g_{j}(0) \neq 0$. In this basis the upper modification at the point $z_{0}$ is represented by the matrix

$$
\Xi^{+}=\left(\begin{array}{cc}
\operatorname{Id}_{N-1} & 0 \\
0 & w
\end{array}\right) .
$$

This is a modification of order 1 , since it increase the degree of $E$

$$
\operatorname{deg}(\widetilde{E})=\operatorname{deg}(E)+\operatorname{deg}\left(\mathcal{O}\left(z_{0}\right)\right)=\operatorname{deg}(E)+1
$$

On the complement to the point $z_{0}$ consider the map

$$
E \stackrel{\Xi^{-}}{\longleftarrow} \widetilde{E}
$$

such that $\Xi^{-} \Xi^{+}=\mathrm{Id}$. It defines the lower modification at the point $z_{0}$. The upper modification $\Xi^{+}$is represented by the vector $(0, \ldots, 1)$ and $\Xi^{-}$by $(0, \ldots,-1)$.

For Higgs bundles the modification acts as

$$
\begin{aligned}
& (E \Phi) \stackrel{\Xi}{\longrightarrow}(\widetilde{E} \widetilde{\Phi}) \\
& \Xi \Phi=\widetilde{\Phi} \Xi, \Xi \widetilde{\bar{A}}=\bar{\partial} \Xi+\bar{A} \Xi .
\end{aligned}
$$

The Higgs fields $\Phi$ and $\widetilde{\Phi}$ should be holomorphic with prescribed simple poles at the marked points. The holomorphity of the Higgs field put restrictions on its form. Consider the upper modification $\Xi^{+} \sim(0, \ldots, 1)$ and assume that $\Phi$ in the above-defined basis takes the form

$$
\Phi=\left(\begin{array}{ll}
a & b \\
c & d
\end{array}\right),
$$

where $a$ is a matrix of order $N-1$. Then

$$
\Xi\left(\begin{array}{ll}
a & b \\
c & d
\end{array}\right)=\left(\begin{array}{cc}
a & b w^{-1} \\
c w & d
\end{array}\right) \Xi .
$$

We see that a generic Higgs field acquires a first order pole after the modification. To escape this, we assume that there exists an eigen-vector $\Phi \xi=\lambda \xi$ such that it belongs to the $\operatorname{Ker} \Phi$. Let $\xi=(0,0, \ldots, 1)$ and

$$
\Phi=\left(\begin{array}{ll}
a & 0 \\
c & d
\end{array}\right) .
$$

Then the Higgs field $\widetilde{\Phi}$ does not have a pole

$$
\widetilde{\Phi}=\left(\begin{array}{cc}
a & 0 \\
c w & d
\end{array}\right) .
$$

In other words the matrix elements $(\Phi)_{j N}$ should have first order null.

In this way the upper modification is lifted from $E$ to the Higgs bundle. After the reduction we come to the map (see (4.17))

$$
T^{*} \mathcal{M}(N, g, n, d) \rightarrow T^{*} \mathcal{M}(N, g, n, d+1) .
$$

We call this the upper Symplectic Hecke Correspondence (SHC).

Generically the modified bundle $\widetilde{E}$ is represented locally as a sum of line bundles $\widetilde{E}=\oplus_{j=1}^{N}\left(\mathcal{L}_{j} \otimes \mathcal{O}\left(z_{0}\right)_{j}^{m}\right)\left(m_{j} \in \mathbb{Z}\right)$ with holomorphic sections

$\widetilde{s}=\left(\widetilde{s}_{1}, \ldots, \widetilde{s}_{N}\right)=\left(w^{-m_{1}} g_{1} s_{1}, w^{-m_{2}} g_{2} s_{2}, \ldots, w^{-m_{N}} g_{N} s_{N}\right)$.

It has the degree

$$
\operatorname{deg}(\widetilde{E})=\operatorname{deg}(E)+\sum_{j=1}^{N} m_{j}
$$

This modification is represented by the vector $\left(m_{1}, \ldots, m_{n}\right)$.

Rememeber that the Higgs field is an endomorphism of $E s \rightarrow \Phi s$ and near $z_{0}$ it acts as

$$
\Phi \cdot s_{j}=(\Phi)_{j}^{k} s_{k}
$$


Similarly the modified Higgs field acts on sections of the modified bundle $\widetilde{E} \widetilde{s} \rightarrow \widetilde{\Phi} \widetilde{s}$. Then it follows from (4.45) that

$$
\widetilde{\Phi} \cdot \widetilde{s}_{j}=\widetilde{\Phi}_{j}^{k} \widetilde{s}_{k}, \widetilde{\Phi}_{j}^{k}=w^{m_{k}-m_{j}} g_{k}(w) g_{j}^{-1}(w) \Phi_{j}^{k} \text {. }
$$

Since $\widetilde{\Phi}$ is holomorphic and $g_{j}(0) \neq 0, \Phi_{j}^{k}\left(z-z_{0}\right)^{m_{k}-m_{j}}$ must be regular at $z=z_{0}$. If we order $m_{1} \geq m_{2} \geq \ldots \geq m_{N}$ then the number of parameters of the endomorphisms is

$\sum_{j<k}\left(m_{j}-m_{k}\right)$. In general case

$$
T^{*} \mathcal{M}(N, g, n, d) \rightarrow T^{*} \mathcal{M}\left(N, g, n, d+\sum_{j=1}^{N} m_{j}\right)
$$

If $\sum_{j=1}^{N} m_{j}=0$ the SHC does not change the topological type of the bundle. Therefore, such SHC defines a Bcklund transformation of integrable hierarchy.

\subsection{Symplectic Hecke correspondence}

$$
\mathcal{R}^{C M} \rightarrow \mathcal{R}^{E T}[11]
$$

We work directly with the Lax matrices

$$
L^{E T} \times \Xi=\Xi \times L^{C M}
$$

The modification matrix should intertwine the multipliers corresponding to the fundamental cycles

$$
\begin{aligned}
& \Xi(z+1, \tau)=Q \times \Xi(z, \tau), \\
& \Xi(z+\tau, \tau)=\widetilde{\Lambda}(z, \tau) \times \Xi(z, \tau) \times \operatorname{diag}\left(\mathbf{e}\left(u_{j}\right)\right) .
\end{aligned}
$$

Consider the modification at $z=0$. The Lax matrix of the CMS has the first order pole

$$
L^{C M} \sim \frac{1}{z} v J .
$$

Its residue has an eigen-vector $\xi^{t}=(1, \ldots, 1)$ with the eigen-value $N-1$. The matrix $\Xi$ satisfying (4.46) and (4.47) that annihilates the vector $\xi$ has the form

$$
\begin{aligned}
& \Xi(z)=\widetilde{\Xi}(z) \times \operatorname{diag}\left((-1) \prod_{j<k ; j, k \neq l}^{l} \theta\left(u_{k}-u_{j}, \tau\right)\right) \\
& \widetilde{\Xi}_{i j}\left(z, u_{1}, \ldots, u_{N}, \tau\right)=\theta\left[\begin{array}{c}
\frac{i}{N}-\frac{1}{2} \\
\frac{N}{2}
\end{array}\right]\left(z-N u_{j}, N \tau\right) .
\end{aligned}
$$

Here $\theta\left[\begin{array}{c}\frac{i}{N}-\frac{1}{2} \\ \frac{N}{2}\end{array}\right]\left(z-N u_{j}, N \tau\right)$ is the theta-function with a characteristic. The determinant of $\Xi$ can be calculated explicitly

$$
\operatorname{det}\left[\frac{\widetilde{\Xi}_{i j}\left(z, u_{1}, \ldots, u_{N}, \tau\right)}{i \eta(\tau)}\right]=\frac{\theta(z)}{i \eta(\tau)} \prod_{1 \leq k<l \leq N} \frac{\theta\left(u_{l}-u_{k}\right)}{i \eta(\tau)},
$$

where $\eta(\tau)=q^{\frac{1}{24}} \prod_{n>0}\left(1-q^{n}\right)$ is the Dedekind function. It has a simple pole at $z=0$ and therefore $\Xi$ is degenerate.

We use the modification to write down the interrelations between the coordinates and momenta of the Calogero-Moser particles and the orbit variables of the Elliptic Top in the $\operatorname{SL}(2, \mathbb{C})$ case

$$
\begin{aligned}
& S_{1}=-v \frac{\theta_{10}(0)}{\theta^{\prime}(0)} \frac{\theta_{10}(2 u)}{\theta(2 u)}-v \frac{\theta_{10}^{2}(0)}{\theta_{00}(0) \theta_{01}(0)} \frac{\theta_{00}(2 u) \theta_{01}(2 u)}{\theta^{2}(2 u)} \\
& S_{2}=-v \frac{\theta_{00}(0)}{\theta^{\prime}(0)} \frac{\theta_{00}(2 u)}{\theta(2 u)}-v \frac{\theta_{00}^{2}(0)}{\theta_{10}(0) \theta_{01}(0)} \frac{\theta_{10}(2 u) \theta_{01}(2 u)}{\theta^{2}(2 u)} \\
& S_{3}=-v \frac{\theta_{01}(0)}{\theta^{\prime}(0)} \frac{\theta_{01}(2 u)}{\theta(2 u)}-v \frac{\theta_{01}^{2}(0)}{\theta_{00}(0) \theta_{10}(0)} \frac{\theta_{00}(2 u) \theta_{10}(2 u)}{\theta^{2}(2 u)} \\
& )
\end{aligned}
$$

Here

$\theta_{1,0}=\sum_{n \in \mathbb{Z}} q^{\frac{1}{2} n^{2}} \exp \pi(2 n-1) z$,

$\theta_{0,0}=\sum_{n \in \mathbb{Z}} q^{\frac{1}{2}\left(n-\frac{1}{2}\right)^{2}} \exp 2 \pi n z$,

$\theta_{0,1}=\sum_{n \in \mathbb{Z}}(-1)^{n} q^{\frac{1}{2}\left(n-\frac{1}{2}\right)^{2}} \exp 2 \pi n z$.

These relations describe the Darboux coordinates $(v, u) \in \mathbb{C}^{2}$ the coadjoint $\mathrm{SL}(2, \mathbb{C})$-orbit $\sum S_{\alpha}^{2}=v^{2}$

It turns out that this modification is equivalent to the twist of $R$-matrices. Namely, it describes the passage from the $\mathrm{dy}$ namical $R$ matrix of the IRF models to the vertex $R$-matrix $[25,26]$. We do not discuss this aspect of SHC here.

\section{$54 d$ theories}

\subsection{Self-dual YM equations and Hitchin equations}

\subsubsection{2-d self-dual equations}

Consider a rank $N$ complex vector bundle $E$ over $\mathbb{R}^{4}$ with coordinates $\mathbf{x}=\left(x_{1}, x_{2}, x_{3}, x_{4}\right)$. Assume that the space of sections is equipped with a nondegenerate Hermitian metric $h,\left(h^{+}=h\right)$. It satisfies the following condition $d h(x, y)=h(\nabla x, y)+h(x, \nabla y)$, where $\nabla$ is a connection on $E$. If $d h(x, y)=0$ for vectors in fibers $y \in V, x \in \widetilde{V}^{t}$, then there exist connections $\nabla_{j}=\partial_{x_{j}}+A_{j}$ such that

$$
\mathbf{A}+=-h^{-1} d h-h^{-1} \mathbf{A} h,\left(\mathbf{A}=\sum_{j=0}^{3} A_{j} d x_{j}\right) .
$$

In this situation the transition functions are reduced to the unitary group $\mathrm{SU}(N) \subset \mathrm{GL}(N, \mathbb{C})$.

Let $F(\mathbf{A}) \in \Omega^{(2)}\left(\mathbb{R}^{4}, s u(N)\right)$ be the curvature $F i j=\left[\nabla_{i}, \nabla_{j}\right]$ or $F(\mathbf{A})=d \mathbf{A}+\mathbf{A}^{2}$. Here $\operatorname{su}(N)=\left\{x \mid x^{+}=-h^{-1} x h\right\}$.

The self-duality equation

$$
F=* F \text {, }
$$

where $*$ is the Hodge operator in $\mathbb{R}^{4}$ takes the form

$$
\left\{\begin{array}{l}
F_{01}=F_{23} \\
F_{02}=F_{31} \\
F_{03}=F_{12}
\end{array}\right.
$$


Assume that $A_{j}$ depend only on $\left(x_{1}, x_{2}\right)$. This means that the fields are invariant under the shifts in directions $x_{0}, x_{3}$. Then $\left(A_{0}, A_{3}\right)$ become adjoint-valued scalar fields which we denote as $\left(\phi_{1}, \phi_{2}\right)$. They are called the Higgs fields. In fact, they will be associated below with the Higgs field $\Phi$. In this way we come to the self-dual equations on the plane $\mathbb{R}^{2}=\left(x_{1}, x_{2}\right)$

$$
\begin{aligned}
& F_{12}=\left[\phi_{1}, \phi_{2}\right], \\
& {\left[\nabla_{1}, \phi_{1}\right]=\left[\phi_{2}, \nabla_{2}\right],} \\
& {\left[\nabla_{1}, \phi_{2}\right]=\left[\nabla_{2}, \phi_{1}\right] .}
\end{aligned}
$$

Introduce complex coordinates $z=x_{1}+i x_{2}, \bar{z}=x_{1}-i x_{2}$ and let $d^{\prime}=\nabla_{z}, d^{\prime \prime}=\nabla_{\bar{z}}$. Consider the fields, taking values in the Lie algebra $\operatorname{sl}(N, \mathbb{C})$

$$
\left\{\begin{array}{l}
\Phi_{z}=\frac{1}{2}\left(\phi_{1}-i \phi_{2}\right) d z \in \Omega^{(1,0)}\left(\mathbb{R}^{2}, \text { ad } \mathrm{E}\right), \\
\Phi_{\bar{z}}=\frac{1}{2}\left(\phi_{1}+i \phi_{2}\right) d \bar{z} \in \Omega^{(0,1)}\left(\mathbb{R}^{2}, \operatorname{ad} \mathrm{E}\right) .
\end{array}\right.
$$

They are not independent since the Hermitian conjugation acts as

$$
\Phi_{z}^{+}=-h^{-1} \Phi_{z} h
$$

Similarly,

$$
\begin{aligned}
& \left\{\begin{array}{l}
A_{z}=\frac{1}{2}\left(A_{1}-i A_{2}\right) \\
A_{\bar{z}}=\frac{1}{2}\left(A_{1}+i A_{2}\right)
\end{array}\right. \\
& A_{\bar{z}}^{+}=-h^{-1} d h-h^{-1} A_{z} h .
\end{aligned}
$$

In terms of fields

$$
\mathcal{W}=\left(A, A_{\bar{z}}, F_{z}, F_{\bar{z}}\right)
$$

$(5.2)-(5.4)$ can be rewritten in the coordinate invariant way:

$$
\begin{cases}\text { 1. } & F+\left[\Phi_{z}, \Phi_{\bar{z}}\right]=0 \\ \text { 2. } & d^{\prime \prime} \Phi_{z}=0 \\ \text { 3. } & d^{\prime} \Phi_{\bar{z}}=0\end{cases}
$$

where $\left[\Phi_{z}, \Phi_{\bar{z}}\right]=\Phi_{z} \Phi_{\bar{z}}+\Phi_{\bar{z}} \Phi_{z}$. Due to (5.5) and (5.6) the third equation is not independent. Thus, we have two equations with the left side of type $(1,1)$ for two complex valued fields $\left(\Phi_{z} A_{\bar{z}}\right)$ and the hermitian matrix $h$.

Equations (5.8) are conformal invariant and thereby can be defined on a complex curve $\Sigma_{g}$. In this case

$$
\begin{aligned}
& \Phi_{z} \in \Omega^{(1,0)}\left(\Sigma_{g}, \operatorname{su}(N)\right), \Phi_{\bar{z}} \in \Omega^{(0,1)}\left(\Sigma_{g}, \operatorname{su}(N)\right), \\
& d^{\prime \prime}: \Omega^{(j, k)}\left(\Sigma_{g}, \operatorname{su}(N)\right) \rightarrow \Omega^{(j, k+1)}\left(\Sigma_{g}, \operatorname{su}(N)\right) .
\end{aligned}
$$

The self-duality equations (5.9) on $\Sigma_{g}$ are called the Hitchin equations.

In fact, instead of (5.8) we will consider further a modified system

$$
\begin{cases}\text { 1. } & F-\left[\Phi_{z}, \Phi_{\bar{z}}\right]=0 \\ \text { 2. } & d^{\prime \prime} \Phi_{z}=0 \\ \text { 3. } & d^{\prime} \Phi_{\bar{z}}=0\end{cases}
$$

This comes from the self-duality on $\mathbb{R}^{4}$ with a metric of signature $(2,2)$. Consider the gauge group action on solutions of (5.9)

$$
\begin{aligned}
& \mathcal{G}=\left\{f \in \Omega^{0}\left(\Sigma_{g}, \mathrm{SU}(N)\right)\right\}, \\
& \Phi_{z} \rightarrow f^{-1} \Phi_{z} f, \Phi_{\bar{z}} \rightarrow f^{-1} \Phi_{\bar{z}} f, \\
& d^{\prime \prime} \rightarrow f^{-1} d^{\prime \prime} f .
\end{aligned}
$$

If $\left(A, A_{\bar{z}}, \Phi_{z}, \Phi_{\bar{z}}\right)$ are solutions of (5.9), then the transformed fields are also solutions. If $f$ takes values in $\operatorname{GL}(N, \mathbb{C})$ then it again transforms solutions to solutions. As above we denote this gauge group as $\mathcal{G}_{\mathbb{C}}$.

Define the moduli space of solutions of (5.9) as a quotient under the gauge group action

$$
\mathcal{M}_{H}\left(S_{g}\right)=\text { solutions of }(5.9) / \mathcal{G} \text {. }
$$

Now look at the second equation in (5.9). It is the moment constraint equation for the Higgs bundles in the absence of marked points (4.14). The gauge group $\mathcal{G}_{\mathbb{C}}$ transforms solutions of (5.9) to solutions but breaks (5.5), (5.6). Now we will restrict ourself with the second equation in (5.9). Dividing the space of its solution on the gauge group $\mathcal{G}_{\mathbb{C}}$ we come to the moduli space of the Higgs bundles $T^{*} \mathcal{M}(N, g, 0, d)(4.17)$. There exists a dense subset of moduli space of stable Higgs bundles $T^{*} \mathcal{M}(N, g, 0, d)^{\text {stable }} \subset T^{*} \mathcal{M}(N, g, 0, d)$. The moduli space of stable Higgs bundles parameterize the smooth part of $\mathcal{M}_{H}\left(\Sigma_{g}\right)(5.13)$ [2].

Consider a Higgs bundle with data $(\Phi, \bar{A})$ satisfying eq. 2 in (5.9) and reconstruct from it solutions $\left(A_{z}, \Phi_{z}, A_{\bar{z}}, \Phi_{\bar{z}}\right)$ of (5.9). Define them as

$$
\begin{aligned}
& \Phi_{z}=\Phi, \Phi_{\bar{z}}=-h^{-1} \Phi^{+} h, \\
& A_{\bar{z}}=\bar{A}, A_{z}=-h^{-1} \bar{\partial} h-h^{-1} \bar{A}^{+} h .
\end{aligned}
$$

Then $\left(\Phi_{\bar{z}}, A_{z}\right)$ satisfy eq. 3. (5.9). Equation 1. (5.9) takes the form

$$
\begin{gathered}
\bar{\partial}\left(h^{-1} \bar{\partial} h+h^{-1} \bar{A}^{+} h\right)-\partial \bar{A}+\left[\bar{A},\left(h^{-1} \bar{\partial} h+h^{-1} \bar{A}^{+} h\right)\right] \\
-\left[\Phi, h^{-1} \Phi^{+} h\right]=0 .
\end{gathered}
$$

For almost all $(\Phi, \bar{A})$ there exists a solution $h$ of this equation (see appendix of Donaldson in [2]). In this way we pass from the holomorphic data to solutions of the system (5.9).

Summarizing, to define $\mathcal{M}_{H}\left(\Sigma_{g}\right)$ one can acts in two ways:

1. Divide the space of solutions of (5.9) on the $\mathrm{SU}(N)$-valued gauge group $\mathcal{G}$.

2. Consider the moduli space of stable Higgs bundles.

\subsubsection{Hyper-Kahler reduction}

In this section we explain how to derive the moduli space $\mathcal{M}_{H}\left(\Sigma_{g}\right)$ (5.13) via an analog of the symplectic reduction. This is the so-called Hyper-Kahler reduction [41]. We prove that infinite-dimensional space $\mathcal{W}(5.7)$ is a Hyper-Kahler 
manifold, and $\mathcal{M}_{H}$ is its Hyper-Kahler quotient, where (5.9) play the role of the moment equations.

To define a Hyper-Kahler manifold we need three complex structures and a metric satisfying certain axioms. Define a flat metric on $\mathcal{W}$ depending on the complex structure on $\Sigma$

$$
\begin{array}{r}
d s^{2}=-\frac{1}{4 \pi} \int_{\Sigma} \operatorname{Tr}\left(\delta A_{z} \otimes \delta A_{\bar{z}}+\delta A_{\bar{z}} \otimes \delta A_{z}\right. \\
\left.+\delta \Phi_{z} \otimes \delta \Phi_{\bar{z}}+\delta \Phi_{\bar{z}} \otimes \delta \Phi_{z}\right) .
\end{array}
$$

Introduce three complex structures $I, J, K$ on $\mathcal{W}$. The corresponding operators act on the tangent bundle $T \mathcal{W}$, such that they obey the imaginary quaternion relations $I^{2}=J^{2}=K^{2}=-I d, I J=K, \ldots$. The complex structures are integrable because $\mathcal{W}$ is flat. Introduce a basis of one-forms in $T^{*} \mathcal{W}$

$$
V=\left(\delta A_{\bar{z}}, \delta \Phi_{z}, \delta A_{z}, \delta \Phi_{\bar{z}}\right)
$$

Then the action of the conjugated operators on $T^{*} \mathcal{W}$ in this basis takes the form

$$
\begin{aligned}
I^{T} & =\left(\begin{array}{cccc}
i & 0 & 0 & 0 \\
0 & i & 0 & 0 \\
0 & 0 & -i & 0 \\
0 & 0 & 0 & -i
\end{array}\right), \\
J^{T} & =\left(\begin{array}{cccc}
0 & 0 & 0 & -1 \\
0 & 0 & 1 & 0 \\
0 & -1 & 0 & 0 \\
1 & 0 & 0 & 0
\end{array}\right), \\
K^{T} & =\left(\begin{array}{cccc}
0 & 0 & 0 & -i \\
0 & 0 & i & 0 \\
0 & i & 0 & 0 \\
-i & 0 & 0 & 0
\end{array}\right) .
\end{aligned}
$$

Linear functions on $\mathcal{W}$ are holomorphic with respect to a complex structure, if they are transformed under the action of the corresponding operator with eigen-value $+i$. Thus $A_{z}$, $\Phi_{z}$ are holomorphic in the complex structure $I, A_{\bar{z}}+i \Phi_{\bar{z}}$, $A_{z}+\Phi_{z}$ are holomorphic in the complex structure $J$, and $A_{\bar{z}}-\Phi_{\bar{z}}, A_{z}+\Phi_{z}$ are holomorphic in the complex structure K.

To be hyper-Kahler on $\mathcal{W}$ the metric $d s^{2}$ should be of type $(1,1)$ in each complex structure. This means that

$d s^{2} \sim\left(I^{T} \otimes I^{T}\right) d s^{2}=\left(J^{T} \otimes J^{T}\right) d s^{2}=\left(K^{T} \otimes K^{T}\right) d s^{2}$.

In this way we have described a flat hyper-Kahler metric on $\mathcal{W}$. A linear combination of the complex structures produces a family of complex structures, parameterized by $\mathbb{C P}^{1}$.

We define three symplectic structures associated with the complex structures on $\mathcal{W}$ as

$\omega_{I}=\left(I^{T} \otimes I d\right) d s^{2}, \omega_{J}=\left(J^{T} \otimes I d\right) d s^{2}, \omega_{K}=\left(K^{T} \otimes I d\right) d s^{2}$.

$$
\begin{aligned}
& \omega_{I}=-\frac{i}{2 \pi} \int_{\Sigma_{g}} \operatorname{tr}\left(D A_{\bar{z}} \wedge D A_{z}-D \Phi_{z} \wedge D \Phi_{\bar{z}}\right), \\
& \omega_{J}=\frac{1}{2 \pi} \int_{\Sigma_{g}} \operatorname{tr}\left(D \Phi_{\bar{z}} \wedge D A_{z}+D \Phi_{z} \wedge \delta A_{\bar{z}}\right), \\
& \omega_{K}=\frac{i}{2 \pi} \int_{\Sigma_{g}} \operatorname{tr}\left(D \Phi_{\bar{z}} \wedge D A_{z}-D \Phi_{z} \wedge D A_{\bar{z}}\right) .
\end{aligned}
$$

These forms are closed and of type $(1,1)$ with respect to the corresponding complex structures.

Now consider the gauge transformations (5.10) of the fields (5.11), (5.12). Since the gauge transform takes values in $\mathrm{SU}(N)$, the forms (5.15) are gauge invariant. Therefore we can proceed as in the case of standard symplectic reduction (3.14). But now we obtain three generating momentum Hamiltonians with respect to the three symplectic forms

$$
\begin{gathered}
F_{I}=\frac{i}{2 \pi} \int_{\Sigma_{g}} \operatorname{tr}\left(\epsilon\left(F_{z, \bar{z}}-\left[\Phi_{z}, \Phi_{\bar{z}}\right]\right)\right),(\epsilon \in \operatorname{Lie}(\mathcal{G})), \\
F_{J}=-\frac{1}{2 \pi} \int_{\Sigma_{g}} \operatorname{tr}\left(\epsilon\left(d^{\prime} \Phi_{\bar{z}}+d^{\prime \prime} \Phi_{z}\right)\right) \\
F_{K}=-\frac{i}{2 \pi} \int_{\Sigma_{g}} \operatorname{tr}\left(\epsilon\left(d^{\prime} \Phi_{\bar{z}}-d^{\prime \prime} \Phi_{z}\right)\right)
\end{gathered}
$$

and the three moment maps $\mathcal{W} \rightarrow \operatorname{Lie}^{*}(\mathcal{G})$

$\mu_{I}=i F-i\left[\Phi_{z}, \Phi_{\bar{z}}\right], \mu_{J}=d^{\prime} \Phi_{\bar{z}}+d^{\prime \prime} \Phi_{z} \mu_{K}=i\left(d^{\prime} \Phi_{\bar{z}}-d^{\prime \prime} \Phi_{z}\right)$.

The zero-valued moments coincide with the Hitchin systems. The hyper-Kahler quotient $\mathcal{W} / / / \mathcal{G}$ is defined as

$$
\mathcal{W} / / / \mathcal{G}=\mu_{I}^{-1}(0) \cap \mu_{J}^{-1}(0) \cap \mu_{K}^{-1}(0) / \mathcal{G}
$$

To come to system (5.9) consider the linear combination

$$
v_{I}=\mu_{J}+i \mu_{K}=d^{\prime \prime} \Phi_{\bar{z}} \text {. }
$$

This moment map is derived from the symplectic form

$$
\Omega_{I}=\omega_{J}+i \omega_{K}=\frac{1}{\pi} \int_{\Sigma} \operatorname{tr}\left(D \Phi_{z} \wedge D A_{\bar{z}}\right) .
$$

This is a (2,0)-form in the complex structure $I$. Thus we have the holomorphic moment map $v_{I}$ in the complex structure $I$. Vanishing of the holomorphic moment map $v_{I}$ and the real moment map $\mu_{I}$ is equivalent to the Hitchin equations. Dividing their solutions on the gauge group $\mathcal{G}$ we come to the moduli space $\mathcal{M}_{H}\left(\Sigma_{g}\right)(5.13)$.

Now consider an analog of (5.16) corresponding to the complex structure $J$

$$
v_{J}=\mu_{K}+i \mu_{I}=\mathcal{F}_{z, \bar{z}}, \mathcal{F}_{z, \bar{z}}=\mathcal{F}\left(\mathcal{A}_{z}, \mathcal{A}_{\bar{z}}\right),
$$

$\mathcal{A}_{z}=A_{z}+i \Phi_{z}, \mathcal{A}_{\bar{z}}=A_{\bar{z}}+i \Phi_{\bar{z}}$. This moment map comes from the symplectic form

$$
\Omega_{J}=\frac{1}{2 \pi} \int_{\Sigma_{g}} \operatorname{tr}(D \mathcal{A} \wedge D \mathcal{A}) .
$$

It is $(2,0)$ form in the complex structure $J$. Putting $v_{J}=0$ we come to the flatness condition of the bundle $E$. Dividing the set of solutions $\mathcal{F}_{z, \bar{z}}=0$ on the $\operatorname{GL}(N, \mathbb{C})$ valued gauge transformations $\mathcal{G}_{\mathbb{C}}$ we come to the space 


$$
\mathcal{Y}=\left(\mathcal{F}_{z, \bar{z}}=0\right) / \mathcal{G}_{\mathbb{C}}
$$

of homomorphisms $\pi_{1}\left(\Sigma_{g}\right) \rightarrow \mathrm{GL}(N, \mathbb{C})$ defined up to conjugations. In accordance with [42] and Donaldson (the appendix in Ref.[2]) generic flat bundles parameterize $\mathcal{M}_{H}\left(\Sigma_{g}\right)$ (5.13) in the complex structure $J$. This space is a phase space of non-autonomous Hamiltonian systems leading to monodromy preserving equations (see Section 6.3). Thus, the space $\mathcal{M}_{H}\left(\Sigma_{g}\right)$ describes phase spaces of integrable systems $\mathcal{R}^{\text {red }}$ (4.17) in the complex structure $I$ and phase spaces of monodromy preserving equations $\mathcal{Y}(5.17)$ in the complex structure $J$.

\section{$5.2 \mathcal{N}=4$ SUSY Yang-Mills in four dimension and Hitchin equations}

Here we consider a twisted version of $\mathcal{N}=4$ super Yang-Mills theory in four dimension. This theory was analyzed in detail in $[16,17,18]$ to develop a field-theoretical approach to the Geometric Langlands Program. Quantum Hitchin systems are one side of this construction and here we use only a minor part of [16]. The twisted theory is a topological theory that contains a generalization of the Hitchin equations (5.9) as a condition of BRST invariance. Our goal is to describe the Hecke transformations in terms of the theory. In section 4 we have defined the Hecke transformations as an instant singular gauge transformation. The four-dimensional theory allows to consider gauge transformations varying along a space coordinate $x_{3}$. They become singular at some point, say $x_{3}=0$, where a singular t'Hooft operator is located. This gives a natural description of the symplectic Hecke correspondence in terms of a monopole configuration in the twisted theory.

\subsubsection{Twisting of SUSY Yang-Mills theory}

$\mathcal{N}=4$ SUSY $\mathrm{SU}(N)$ Yang-Mills action in four dimension can be derived from the $\mathcal{N}=4$ SUSY SU $(N)$ Yang-Mills action in ten dimensions by dimensional reduction. We need only the bosonic part of the reduced theory.

The bosonic fields of the $4 \mathrm{~d}$ Yang-Mills theory are the four-dimensional gauge potential

$$
A=\left(A_{0}, A_{1}, A_{2}, A_{3}\right),
$$

and six scalar fields coming from six extra dimensions

$$
\phi=\left(\phi_{0}, \phi_{1}, \phi_{2}, \phi_{3}, \phi_{4}, \phi_{5}\right) \text {. }
$$

The bosonic part of the action has the form

$$
\begin{aligned}
I=\frac{1}{e^{2}} \int d^{4} x \operatorname{tr} & \left(\frac{1}{2} \sum_{\mu, v=0}^{3} F_{\mu \nu} F^{\mu v}+\sum_{\mu=0}^{3} \sum_{\imath=1}^{6} D_{\mu} \phi_{i} D^{\mu} \phi^{i}\right. \\
& \left.\frac{1}{2} \sum_{i, j=0}^{6}\left[\phi_{i} \phi_{j}\right]^{2}\right) .
\end{aligned}
$$

The symmetry of the action is $\operatorname{Spin}(4) \times \operatorname{Spin}(6)$

(or $\operatorname{Spin}(1,3) \times \operatorname{Spin}(6)$ in the Lorentz signature). The sixteen generators of the $4 \mathrm{~d}$ supersymmetry are transformed under $\operatorname{Spin}(1,3) \times \operatorname{Spin}(6) \sim S L(2) \times S L(2) \times \operatorname{Spin}(6)$ as $(2,1,4) \oplus(1,2,4)$ :
$\left\{\bar{Q}_{A X}\right\} \oplus\left\{Q_{A}^{Y}\right\},(A=1,2 ; X=1, \ldots, 4),(\dot{A}=1,2 ; Y=1, \ldots, 4)$.

They satisfy the super-symmetry algebra

$\left\{\bar{Q}_{A X}, Q_{A}^{Y}\right\}=\delta_{X}^{Y} \sum_{\mu=0}^{3} \Gamma_{A \dot{A}}^{\mu} P_{\mu},\{Q, Q\}=0,\{\bar{Q}, \bar{Q}\}=0$.

The action of $Q$ on a field $X$ takes the form

$$
\delta X=\{Q, X\} .
$$

Let $\kappa$ be a map $\operatorname{Spin}(4) \rightarrow \operatorname{Spin}(6)$ and set

$$
\operatorname{Spin}^{\prime}(4)=(I d \times \kappa) \operatorname{Spin}(4) .
$$

Define $\kappa$ in such a way that the action of $\operatorname{Spin}^{\prime}(4)$ on the chiral spinor $\mathcal{S}^{+}$has an invariant vector. Let $Q$ be the corresponding supersymmetry. It follows from (5.18) that it obeys $Q^{2}=0$. The twisted theory is defined by the physical observables from the cohomology groups $H^{\bullet}(Q)$. The twisted four scalar fields $\phi=\left(\phi_{0}, \ldots, \phi_{3}\right)$ are reinterpreted as adjoint-valued one-forms on $\mathbb{R}^{4}$, while untwisted $\sigma, \bar{\sigma}=\phi_{4} \pm i \phi_{5}$ remain adjoint-valued scalars.

In fact there is a family of topological theories parameterized by $t \in \mathbb{C P}^{1}$. To be invariant under $Q$ the bosonic fields should satisfy the equations

$$
\begin{aligned}
& \text { 1) } \quad(F-\phi \wedge \phi+t D \phi)^{+}=0, \\
& \text { 2) } \quad\left(F-\phi \wedge \phi-t^{-1} D \phi\right)^{-}=0, \\
& \text { 3) } \quad * D * \phi=0,
\end{aligned}
$$

where \pm denote the self-dual and the anti-self-dual parts for four-dimensional two-forms, $D=d+[\mathbf{A}$, ] and $*$ is the Hodge operator in four dimension. We are interested in solutions of this system up to gauge transformations.

This theory defined on flat $\mathbb{R}^{4}$ can be extended to any four-manifold $M$ in such a way that it preserves the $Q$-symmetry and the contributions of the metric come only from $Q$-exact terms. The bosonic part of the theory is described by connections $\mathbf{A}=\left(A_{0}, A_{1}, A_{2}, A_{3}\right)$ in a bundle $E$ over $M$ in the presence of the adjoint-valued one-forms $\phi=\left(\phi_{0}, \phi_{1}, \phi_{2}, \phi_{3}\right)$ satisfying (5.19).

The important thing for the case of integrable systems is $M=\mathbb{R}^{2} \times \Sigma_{g}$, where $\mathbb{R}^{2}=\left(\right.$ time $\left.=x_{0}\right) \times\left(x_{3}=y\right)$ and $\Sigma_{g}$ will play the role of the basic spectral curve. $\mathbb{R}^{2}$ is not involved in the twisting and the fields $\left(\phi_{0}, \phi_{3}\right)$ remain scalars, while $\phi_{1}, \phi_{2}$ become one-forms on $\Sigma_{g}$. It turns out that after reduction the system (5.19) becomes equivalent to the Hitchin equations (5.9).

\subsubsection{Hecke correspondence and monopoles}

The system (5.19) for $t=1$ can be replaced by

$$
\begin{aligned}
& F-\phi \times \phi+* D \phi=0 \\
& * D * \phi=0
\end{aligned}
$$

Assume that the fields are time independent and consider the system on the three-dimensional manifold $W=I\left(x_{3}\right) \times \Sigma_{g}$, where $-\infty \leq x_{3} \leq \infty$. In terms of the tree-dimensional fields 
and $\widetilde{\Phi}\left(\mathbf{A}=\left(A_{0}, \widetilde{A}\right), \phi=\left(\Phi_{0} d x_{0}, \widetilde{\Phi}\right)\right.$, the equations take the form [16]

$$
\begin{aligned}
& \widetilde{F}-\widetilde{\Phi} \wedge \widetilde{\Phi}=*\left(D \Phi_{0}-\left[A_{0}, \widetilde{\Phi}\right]\right), \\
& * D \widetilde{\Phi}=\left[\Phi_{0}, \widetilde{\Phi}\right]+D A_{0}, \\
& * D * \widetilde{\Phi}+\left[A_{0}, \Phi_{0}\right]=0 .
\end{aligned}
$$

Here the Hodge operator $*$ is taken in the three-dimensional sense. Replace the coordinates $\vec{x}=\left(x_{1}, x_{2}, x_{3}\right)$ on $x_{3} \rightarrow y$ and $\left(x_{2}, x_{3}\right) \rightarrow(z, \bar{z})$, where $(z, \bar{z})$ are local coordinates on $\Sigma_{g}$. Let $g(z, \bar{z})|d z|^{2}$ be a metric on $\Sigma_{g}$. The metric on $W$ is

$$
d s^{2}=g|d z|^{2}+d y^{2} .
$$

Then the Hodge operator takes the form $* d y=\frac{1}{2} i g d z \wedge d \bar{z}, \quad * d z=-i d z \wedge d y, \quad * d \bar{z}=i d \bar{z} \wedge d y$.

It is argued in [16] that $\Phi_{y}=0$ and $A_{0}=0$ are solutions of the system. Then we come to the equations

$$
\begin{aligned}
& \text { 1. } F\left(A_{z}, A_{\bar{z}}\right)-\left[\Phi_{z}, \Phi_{\bar{z}}\right]=\frac{1}{2} i g D_{A_{y}} \Phi_{0} \text {, } \\
& \text { 2. } D A_{\bar{z}} \Phi_{z}=0, \\
& \text { 3. } F\left(A_{y}, A_{\bar{z}}\right)=i D_{A_{\bar{z}}} \Phi_{0}, \\
& \text { 4. } D_{A_{y}} \Phi_{z}=-i\left[\Phi_{z}, \Phi_{0}\right] .
\end{aligned}
$$

where as before $\Phi_{z}^{+}=-h^{-1} \Phi_{\bar{z}} h$. The system is simplified in the gauge $A_{y}=0$. In particular, for $\Phi_{0}=0$ the system (5.23) becomes essentially two-dimensional and coincides with the Hitchin equations (5.9).

Let $\Sigma_{g}$ be an elliptic curve $(g=1)$. This case is important for application to integrable systems. The nonlinear system (5.23) can be rewritten as a compatibility condition for the linear system depending on the spectral parameter $\lambda \in \mathbb{C}$

$$
\left\{\begin{array}{l}
\left(\partial_{z}+\lambda^{-1} a \partial_{y}+A_{z}+i \lambda^{2} \Phi_{z}+i \lambda^{-1} a \Phi_{0}\right) \psi=0, \\
\left(\partial_{\bar{z}}+\lambda a \partial_{y}+A_{z}+i \lambda^{-2} \Phi_{\bar{z}}-i \lambda a \Phi_{0}\right) \psi=0
\end{array}\right.
$$

Here $a^{2}=\frac{1}{\bar{\tau}-\tau}$. This linear system allows to apply the methods of the Inverse Scattering Problem or the Whitham approximation to find solutions of (5.23).

Define the complex connection $\mathcal{A}_{z}=A_{z}+i \Phi_{z}$. In terms of $\left(\mathcal{A}_{z}, \mathcal{A}_{\bar{z}}, A_{y}\right)$ the systems (5.23) assumes the form of the Bogomolny equation

$$
F=* D \Phi_{0} .
$$

Consider a monopole solution of this equation. Let

$$
\widetilde{W}=\left(W \backslash \vec{x}^{0}=\left(y=0, z=z_{0}\right)\right) .
$$

The Bianchi identity $D F=0$ in the space $\widetilde{W}$ implies that $\Phi_{0}$ is the Green function for the operator $* D *$

$$
* D * D \Phi_{0}=M \delta\left(\vec{x}-\vec{x}^{0}\right),
$$

where $M \in \operatorname{gl}(N, \mathbb{C})$.
Consider first the abelian case $G=\mathrm{U}(1)$. Then $F\left(A_{z}, A_{\bar{z}}\right)$ is a curvature of a line bundle $\mathcal{L}$. Locally near $\vec{x}^{0}=\left(y=0, z=z_{0}, \bar{z}=\bar{z}_{0}\right) \Phi_{0}$ has a singularity

$$
\Phi_{0} \sim \frac{i m}{2\left|\vec{x}-\vec{x}^{0}\right|},
$$

where $m$ is a magnetic charge. Due to 1 . in (5.23), $F$ takes the form

$$
\begin{aligned}
& F\left(A_{z}, A_{\bar{z}}\right)=\frac{1}{2} g \partial_{y} \Phi_{0}, \\
& F\left(A_{z}, A_{\bar{z}}\right) \sim \frac{1}{2} m g(z, \bar{z}) \frac{y}{\left|\vec{x}-\vec{x}^{0}\right|^{\frac{3}{2}}} .
\end{aligned}
$$

Consider a small sphere $S^{2}$ enclosing $\vec{x}^{0}$. Due to (5.24) and (5.25)

$$
\int_{S^{2}} F=m \text {. }
$$

This solution describes the Dirac monopole of charge $m$ corresponding to a line bundle over $S^{2}$ of degree $m$.

Let $\Sigma_{g}^{ \pm}=\Sigma_{g} \times( \pm \infty)$ and $\mathcal{L}^{ \pm}$be the line bundles over $\Sigma_{g}^{ \pm}$. The two-dimensional cycle $C$ describing the boundary

$$
C=\partial\left(\left(W=I \times \Sigma_{g}\right) \backslash \vec{x}^{0}\right) \text { is } \Sigma_{g}^{+}-\Sigma_{g}^{-}-S^{2} .
$$

Taking the integral over $C$ we find that

$$
\int_{C} F=0 \text {. }
$$

In other words, for the Chern classes of the bundles $c(\mathcal{L})=\operatorname{deg}(\mathcal{L})$ we have

$$
\operatorname{deg}\left(\mathcal{L}^{+}\right)=\operatorname{deg}\left(\mathcal{L}^{-}\right)+m
$$

or $\mathcal{L}^{+}=\mathcal{L}^{\otimes} \mathcal{O}\left(z_{0}\right)^{m}$. Here $\mathcal{O}\left(z_{0}\right)^{m}$ is a line bundle whose holomorphic sections are holomorphic functions away from $z_{0}$ with a possible single pole of degree $m$ at $z_{0}$. The line bundles over $\Sigma_{g}$ are topologically equivalent for $y<0$ or $y>0$. The gauge transformation $\Phi_{0}$ is smooth away from $\vec{x}^{0}$. The singularity change the degree of the bundle.

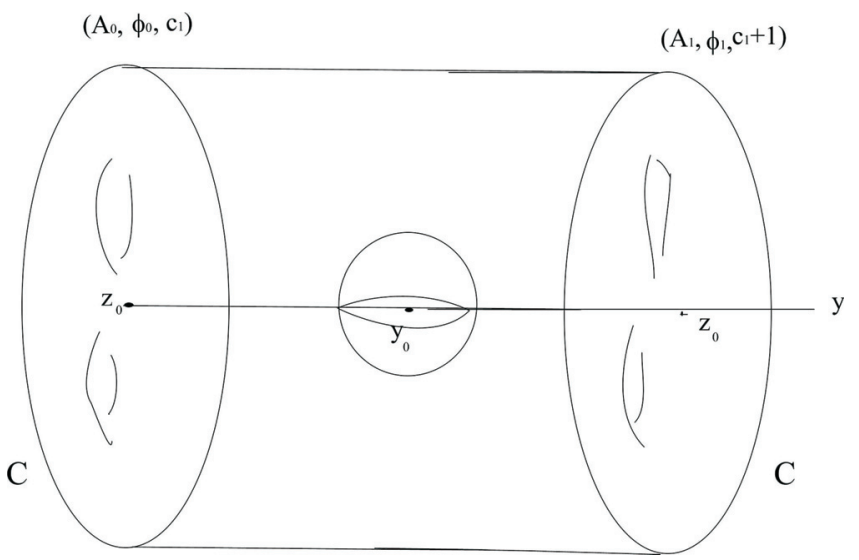

Fig. 3: Monopole, located at $y_{0}$ changes the Chern class 
The monopole increase the Chern class: $c_{1} \rightarrow c_{1}+m$.

In four-dimensional abelian theory we have the Dirac monopole singular along the time-like line $L=\left(x_{0}, \vec{x}^{0}\right)$. This corresponds to including the t'Hooft operator in the theory saying that the connections have monopole singularity along the line $L$.

A generic vector bundles $E$ near $\vec{x}^{0}$ splits

$$
E_{y} \sim \mathcal{L}_{1} \oplus \mathcal{L}_{2} \oplus \ldots \oplus \mathcal{L}_{N}
$$

Consider the gauge transformation

$$
\Phi_{0} \sim \frac{i}{2\left|\vec{x}-\vec{x}^{0}\right|} \operatorname{diag}\left(m_{1}, \ldots, m_{N}\right) .
$$

This causes the transformation $\mathcal{L}_{j} \rightarrow \mathcal{L}_{j} \otimes \mathcal{O}\left(z_{0}\right)^{m_{j}}$. The degree of the bundles $E$ changes after crossing $y=0$ by $\sum m_{j}$, as it was described for bundles over $\Sigma$ in Section 4.4 .

To be more precise we specify the boundary conditions of solutions on the ends $y=-\infty$ and $y=+\infty$. Since $\Phi_{0} \rightarrow 0$ for $y \rightarrow \pm \infty$ system (5.23) coincides with the Hitchin system (5.9). If $\mathcal{M}_{H}\left(N, g, n, m^{ \pm}\right)$is the moduli space of solutions on the boundaries $y= \pm \infty$ the gauge transformation with the monopole singularity stands that $m^{+}=m^{-}+\sum m_{j}$. It defines the SHC between two integrable systems related to $\mathcal{M}_{H}\left(N, g, n, m^{ \pm}\right)$. In particular, we have described it at the point $y=0$ for $\mathcal{M}_{H}(N, 1, n, 0)$ and $\mathcal{M}_{H}(N, 1, n, 1)$.

\section{Conclusion}

Here we will briefly discus some related issues have not included in the lectures.

\section{1}

Solutions of the Hitchin equations (5.9) corresponding to quasi-parabolic Higgs bundles were analyzed in Ref. [17]. In the three-dimensional gauge theory considered in Section 4.3 we have the Wilson lines located at the marked points. In the four-dimensional Yang-Mills theory they corresponds to singular operators along two-dimensional surfaces. Locally on a punctured disc around a marked point the Hitchin system (5.9) assumes the form of the Nahm equations [43]. It was proved in Ref. [46] that the space of its solutions after dividing on a special gauge group is symlectomorphic to a coadjoint orbit of $\operatorname{SL}(N, \mathbb{C})$. A hyper-Kahler structure on the space of solutions induces a hyper-Kahler structure on the orbits. It establishes the interrelations between the Hitchin equations and the Higgs bundles with the marked points (the quasi-parabolic Higgs bundles).

\section{2}

There exists a generalization of this approach to Higgs bundles of infinite rank. In other words, the structure group $G=\mathrm{GL}(N, \mathbb{C})$ or $\operatorname{SL}(N, \mathbb{C})$ of the bundles is replaced by an in- finite-rank group. One way is to consider the central extended loop group $S^{1} \rightarrow G$. Then the Higgs field depends on additional variable $x \in S^{1}$ and instead of the Lax equation we come to the Zakharov-Shabat equation

$$
\partial_{j} L-\partial_{x} M_{j}+\left[M_{j}, L\right]=0 .
$$

This equation describes an infinite-dimensional integrable hierarchy like the KdV hierarchy. The two-dimensional version of ECMS was constructed in [10,11]. In particular, SHC establishes an equivalence of the two-particles $(N=2)$ elliptic Calogero-Moser field theory with the Landau-Lifshitz equation $[44,45]$. The latter system is the two-dimensional version of the $\operatorname{SL}(2, \mathbb{C})$ elliptic top. Relations (4.48) work in the two-dimensional case.

Another way is to consider GL( $\infty)$ bundles. In Ref. [48] ECMS for infinite number of particles $N \rightarrow \infty$ was analyzed. The elliptic top on the group of the non-commutative torus was considered in Ref. [47]. It is a subgroup of $\operatorname{GL}(\infty)$. This construction describes an integrable modification of the hydrodynamics of the ideal fluid on a non-commutative two-dimensional torus.

\section{3}

Consider dynamical systems, where the role of times is played by parameters of complex structures of curves $\Sigma_{g, n}$. In this case we come to monodromy preserving equations, like the Schlesinger system or the Painlevé equations. They can be constructed in the similar fashion as the integrable Hitchin systems [8]. To this purpose the one should replace the Higgs bundles by the flat bundles and afterwards use the same symplectic reduction (see (5.17)). In this situation the Lax equations takes the form

$$
\partial_{j} L-\partial_{z} M_{j}+\left[M_{j}, L\right]=0 .
$$

An analysis of this system is more complicated than the standard Lax equations due to the presence of derivative with respect to the spectral parameter. Note that $M_{j}$ corresponds only to quadratic Hamiltonians, since they responsible for the deformations of complex structures. Concrete examples of this construction was given in $[8,52,53]$. Interrelations with Higgs bundles were analyzed in $[8,49]$. It is remarkable that the Symplectic Hecke correspondence works in this case. It establishes an equivalence of the Painlevé VI equation and a non-autonomous Zhukovski-Volterra gyrostat [12].

\section{4}

A modification of the Higgs bundles allows one to construct relativistic integrable systems [50]. The role of the Higgs field is played by a group element $g=\exp \left(c K^{-1} \Phi\right)$ where $K$ is a canonical class on $\Sigma$ and $c$ is the relativistic parameter. This construction works only for curves of genus 
$g \leq 1$. This approach was realized in Ref. [28] to derive the elliptic Rujesenaars system and in Ref. [51, 53] to derive the elliptic classical r-matrix of Belavin-Drinfeld [54] and a quadratic Poisson algebra of the Sklyanin-Feigin-Odesski type $[55,56]$.

The inclusion of the relativistic systems allows to define a duality in integrable systems [57, 58] (see [59] for recent developments). This type of dualities has a natural description for the corresponding quantum integrable systems in terms of Hecke algebras [60]. There, it is called the Fourier transform and takes the form of $S$-duality. Another form of duality in the classical Hitchin system considered in [61, 62, 63]. It is related to Langlands duality and similar to $T$-duality of fibers in the Hitchin fibration.

\section{5}

There is an useful description of the moduli space of holomorphic vector bundles closely related to the modification described in Section 4.4. It is so-called Tyurin parametrization [64]. This construction was applied to describe Higgs bundles and integrable systems related to a curve of arbitrary genus in Ref. [10, 65, 66]. Using this approach classical r-matrices with a spectral parameter living on curves of arbitrary genus were constructed in Ref. [67].

\section{References}

[1] Hitchin, N.: Stable Bundles and Integrable Systems. Duke Math. Jour. Vol. 54 (1987), p. 91-114.

[2] Hitchin, N.: The Self-Duality Equations on a Riemann surface. Proc. London Math. Soc,. Vol. 55 (1987), 59-126.

[3] Hurtubise, J.: Integrable Systems and Algebraic Surfaces. Duke Math. Journ., Vol. 83 (1996), 19-50.

[4] Gorsky, A., Nekrasov, N., Rubtsov, V.: Hilbert Schemes, Separated Variables, and D-Branes. Commun. Math. Phys., Vol. 222 (2001), p. 299-318.

[5] Markman, E.: Spectral curves and integrable systems. Comp. Math., Vol. 93 (1994), p. 255-290.

[6] Gorsky, A., Nekrasov, N.: Elliptic Calogero-Moser System from two Dimensional Current Algebra. [hep-th/9401021].

[7] Enriquez, B., Rubtsov, V.: Hitchin Systems, Higher Gaudin Operators and R-matrices. Math. Res. Letter, Vol. 3 (1996), p. 343-357.

[8] Levin, A., Olshanetsky, M.: Isomonodromic Deformations and Hitchin Systems. Amer. Math. Soc.Transl. (2), Vol. 191 (1999), p. 223-262.

[9] Nekrasov, N.: Holomorphic Bundles and Many-Body Systems. Commun. Math. Phys., Vol. 180 (1996), p. 587-604, [hep-th/9503157].

[10] Krichever, I.: Vector Bundles and Lax Equations on Algebraic Curves. Commun. Math. Phys., Vol. 229 (2002), p. 229-269, [hep-th/0108110].
[11] Levin, A., Olshanetsky, M., Zotov, A.: Hitchin Systems Symplectic Hecke Correspondence and Two-dimensional Version. Comm. Math. Phys., Vol. 236 (2003), p. 93-133.

[12] Levin, A., Olshanetsky, M., Zotov, A.: Painleve VI, Rigid Tops and Reflection Equation. Commun. Math. Phys., Vol. 268 (2006), p. 67-103.

[13] Gorsky, A., Krichever, I., Marshakov, A., A. Mironov, A., Morozov, A.: Integrability and Seiberg-Witten Exact Solution. Phys. Lett. B, Vol. 355, 466 (1995), [arXiv:hep-th/9505035].

[14] Seiberg, N., Witten, E.: Electric - Magnetic Duality, Monopole Condensation, and Confinement in $N=2 \mathrm{Su}-$ persymmetric Yang-Mills Theory. Nucl. Phys. B, Vol. 426 (1994), p. 19-52 [Erratum-ibid. B Vol. 430, 485(1994)], [arXiv:hep-th/9407087].

Seiberg, N., Witten, E.: Monopoles, Duality and Chiral Symmetry Breaking in $N=2$ Supersymmetric QCD. Nucl. Phys. B, Vol. 431 (1994), p. 484-550

[arXiv:hep-th/9408099].

[15] Gorsky, A., Mironov, A.: Integrable Many-Body Systems and Gauge Theories. [arXiv:hepth/0011197].

[16] Kapustin, A., Witten, E. Electric-Magnetic Duality and the Geometric Langlands Program. [arXiv:hep-th/0604151].

[17] Gukov, S., Witten, E.: Gauge Theory, Ramification, and the Geometric Langlands Program.

[arXiv:hep-th/0612073].

[18] Witten, E.: Gauge Theory and Wild Ramification. [arXiv:hep-th/07100631].

[19] Olshanetsky, M., Perelomov, A.: Classical Integrable Finite-Dimensional Systems Related to Lie Algebras, Physics Reports, Vol. 71 (1981), p. 313-400.

[20] Donagi, R.: Siebrg-Witten Integrable Systems, [arXiv:alg-geom/9705010].

[21] Etingof, P.: Lectures on Calogero-Moser Systems, [arXiv:math/0606233].

[22] Zotov, A.: Classical Integrable Systems and their Field-Theoretical Generalizations, Phys. Part. Nucl. Vol. 37 (2006), p. 400-443; Fiz. Elem. Chast. Atom. Yadra Vol. 37 (2006), p. 759-842.

[23] G. t'Hooft, G.: On the Phase Transition Towards Permanent Quark Cofinement, Nucl. Phys. B, Vol. 138, (1978).

[24] Kapustin, A.: Wilson-'t Hooft Operators in Four-Dimensional Gauge Theories and S-Duality, Phys. Rev. D, Vol. 74 (2006), 025005.

[25] Baxter, R. J.: Eight-Vertex Model in Lattice Statistics and One-Dimensional Anisotropic Heisenberg Chain, I. Ann. Phys., Vol. 76 (1973), p. 48-71.

[26] E. Date, M. Jimbo, T. Miwa, M. Okado, Fusion of the Eight Vertex SOS Model, Lett. Math.Phys., Vol. 12 (1986), p. 209.

[27] Arnold, V.: Mathematical Methods in Classical Mechanics. Springer, 1978. 
[28] Arutyunov, G., Frolov, S., Medvedev, P.: Elliptic Ruijsenaars-Schneider Model from the Cotangent Bundle over the Two-Dimensional Current Group. J. Math. Phys., Vol. 38 (1997), p. 5682-5689.

[29] Simpson, C.: Harmonic Bundles on Noncompact Curves, Journ. Am. Math. Soc., Vol. 3 (1990), p. 713-770.

[30] Faltings, G.: Stable G-Bundles and Projective Connections. J. Alg. Geo., Vol. 2 (1993), p. 507-568.

[31] Donagi, R.: Spectral covers, in Current Topics in Complex Algebraic Geometry. (H. Clemens and J. Kollar, eds., MSRI Pub., Vol. 28 (1995), p. 65-86,

[arXive:alg-geom/9505009].

[32] Calogero, F.: Solution of the One-Dimensional n-Body Problem with Quadratic and/or Inversely Quadratic Pair Potentials.J. Math. Phys., Vol. 12 (1971), p. 419-436; Exactly Solvable One-Dimensional Many-Body Problem. Lett. Nuоvo Cim., Vol. 13 (1975), p. 411.

[33] Moser, J.: Three Integrable Systems Connected with Isospectral Deformations. Adv. Math., Vol. 16 (1975), p. $1-23$.

[34] Gibbons, J., Hermsen, T.: A Generalization of Calogero-Moser System. Physica D, Vol. $11 D$ (1984), p. 337-348.

[35] Olshanetsky, M., Perelomov, A.: Explicit Solution of the Calogero Model in the Classical Case and Geodesic Flows on Symmetric Space of Zero Curvature. Lett. Nuovo Cim. Vol. 16 (1976), p. 333-339.

[36] Kazdan, D., Kostant, B., Sternberg, S.: Hamiltonian Group Actions and Dynamical Systems of Calogero Type. Comm. Pure and Appl. Math., Vol. 31 (1978) p. $481-507$.

[37] Faddeev, L., Slavnov, A.: Gauge Fields: Introduction to Quantum Field Theory. Benjamin Reading, 1980.

[38] Konopleva, N., Popov, V.: Gauge Fields. NM Queen Harwood Academic Publishers New York, 1981.

[39] Krichever, I.: Elliptic Solutions of the Kadomtsev-Petviashily Equation and Many-Body Problems. Funct. Anal. Aplic. Vol. 14 (1980), p. 45.

[40] Reyman, A., Semenov-Tian-Schansky, M.: Lie Algebras and Lax Equations with Spectral Parameter on Elliptic Curve (Russian). Zap. Nauchn. Sem. Leningrad. Otdel. Mat. Inst. Steklov. (LOMI) 150 (1986), Voprosy Kvant. Teor. Polya i Statist. Fiz., Vol. 6 p. 104-118, 221; translation in Journal Soviet Math., Vol. 46 (1989), No. 1, p. 1631-1640.

[41] Hitchin, N., Karlhede, A., Lindström, U., Roček, M.: Hyper-Kähler Metrics and Supersymmetry. Comm. Math. Phys., Vol. 108 (1987), p. 535-589.

[42] Corlette, K.: Flat G-Bundles with Canonical Metrics. J. Diff. Geom., Vol. 28 (1988), p. 361-382.

[43] Nahm, W.: The Construction of All Selfdual Multi-Monopoles Adhm Method, Trieste Cent. Theor. Phys., IC-82-016.

[44] Sklyanin, E.: On Complete Integrability of the Landau-Lifshitz Equation. Preprint LOMI E-3-79 (1979).
[45] Borovik, A., Robuk, V.: Linear Pseudopotentials and Conservation Laws for the Landau-Lifshitz Equation. Theor. Math. Phys., Vol. 46 (1981), p. 371-381.

[46] P. Kronheimer, A Hyper-Kähler Structure on Coadjoint Orbits of a Semisimple Complex Group.J. London Math. Soc., Vol. 42 (1990), p. 193-208.

[47] Khesin, B., Levin, A., Olshanetsky, M.: Bihamiltonian Structures and Quadratic Algebras in Hydrodynamics and on Non-Commutative Torus. Comm. Math. Phys., Vol. 250 (2004), p. 581-612.

[48] Olshanetsky, M.: The Large N Limits of Integrable Models. Mosc. Math. J., Vol. 3 (2003), p. 1307-1331.

[49] Takasaki, K.: Spectral Curves and Whitham Equations in the Isomonodromic Problems of Schlesinger Type. [solv-int/9704004].

[50] Ruijsenaars, S. N. M.: Complete Integrability of Relativistic Calogero-Moser Systems and Elliptic Function Identities. Commun. Math. Phys., Vol. 110:191 (1987); Ruijsenaars, S. N. M., Schneider, H.: A New Class of Integrable Systems and its Relation to Solitons. Ann. Phys., Vol. 170 (1986), 370-405.

[51] Braden, H., Dolgushev, V., Olshanetsky, M., Zotov, A.: Classical r-Matrices and the Feigin-Odesskii Algebra via Hamiltonian and Poisson Reductions. Journ. Phys. A, Vol. 36 (2003), p. 6979-7000.

[52] Takasaki, K.: Gaudin Model, KZ Equation, and Isomonodromic Deformation on Torus. Lett. Math. Phys., Vol. 44 (1998), p. 143-156. [hep-th/9711058].

[53] Chernyakov, Yu., Levin, A., Olshanetsky, M., Zotov, A.: Elliptic Schlesinger System and Painlevé VI. Journ. Phys. A, Vol. 39 (2006), p. 12083-120102.

[54] Belavin, A., Drinfeld, V.: Solutions of the Classical Yang-Baxter Equation for Simple Lie Algebras. Funct. Anal. and Applic., Vol. 16 (1982), No. 3, p. 1-29.

[55] Sklyanin, E.: Some Algebraic Structures Connected with the Yang-Baxter Equation. Funct. Anal. and Applic., Vol. 16 (1982), No. 4, p. 27-34.

[56] Feigin, B., Odesski, A.: Sklyanin's Elliptic Algebras. Funct. Anal. and Applic., Vol. 23 (1989), No. 3, p. 207-214.

[57] Fock, V., Gorsky, A., Nekrasov, N., Rubtsov, V.: Duality in Integrable Systems and Gauge Theories. JHEP 0007 (2000) 028, [arXiv:hep-th/9906235].

[58] Gorsky, A., Rubtsov, V.: Dualities in Integrable Systems: Geometrical Aspects. [arXiv:hepth/0103004].

[59] Abenda, S., Previato, E.: How is the Hitchin System Self-Dual? Let us Count the Ways. Inst. Mittag Lefler, Report No. 64 2006/2007.

[60] Cherednik, I.: Double Affine Hecke Algebras. Cambridge University Press, 2005.

[61] Hausel, T., Thaddeus, M.: Mirror Symmetry, Langlands Duality, and the Hitchin System. Invent. Math., Vol. 153 (2003), p. 197-229.

[62] Donagi, R., Pantev, T.: Langlands Duality for Hitchin Systems. [arXiv:math/0604617]. 
[63] Hitchin, N.: Langlands Duality and G2 Spectral Curves. [arXiv:math/0611524].

[64] Tyurin, A.: Classification of Vector Bundles over an Algebraic Curve of Arbitrary Genus. Amer. Math. Soc. Transl. II Ser. 63 (1967), p. 245- 279.

[65] Enriquez, B., Rubtsov, V.: Hecke-Tyurin Parametrization of the Hitchin and KZB Systems. [arXiv:math/9911087].

[66] Takasaki, K.: Tyurin Parameters of Commuting Pairs and Infinite Dimensional Grassmann Manifold. Contribution to proceedings of RIMS workshop "Elliptic Integrable Systems" (RIMS, 2004). [arXiv:nlin/0505005].

[67] Dolgushev, V.: R-Matrix Structure of Hitchin System in Tyurin Parameterization. Commun. Math. Phys., Vol. 238 (2003), p. 131-147.

Mikhail Olshanetsky

e-mail: olshanet@itep.ru

Chern Institute of Mathematics

Nankai University

Tianjin, China 